Article

\title{
Numerical Investigation of Performance Enhancement of the S809 Airfoil and Phase VI Wind Turbine Blade Using Co-Flow Jet Technology
}

\author{
Shunlei Zhang, Xudong Yang * and Bifeng Song
}

check for updates

Citation: Zhang, S.; Yang, X.; Song, B.

Numerical Investigation of

Performance Enhancement of the S809 Airfoil and Phase VI Wind Turbine Blade Using Co-Flow Jet Technology. Energies 2021, 14, 6933. https://doi.org/10.3390/en14216933

Academic Editor: Andrzej Bielecki

Received: 30 September 2021

Accepted: 20 October 2021

Published: 21 October 2021

Publisher's Note: MDPI stays neutral with regard to jurisdictional claims in published maps and institutional affiliations.

Copyright: (c) 2021 by the authors. Licensee MDPI, Basel, Switzerland. This article is an open access article distributed under the terms and conditions of the Creative Commons Attribution (CC BY) license (https:/ / creativecommons.org/licenses/by/ $4.0 /)$.
National Key Laboratory of Science and Technology on Aerodynamic Design and Research, School of Aeronautics, Northwestern Polytechnical University, Xi'an 710072, China; zhangsl@mail.nwpu.edu.cn (S.Z.); bfsong@nwpu.edu.cn (B.S.)

* Correspondence: xdyang@nwpu.edu.cn

\begin{abstract}
Making full use of wind energy can effectively alleviate the global energy shortage and environment contamination problems. Nevertheless, how to significantly improve the performance of the wind turbine airfoil and blade is a crucial issue. As the novel flow control method, the co-flow jet (CFJ) technology is one of the most potential methods to solve this problem. Thus, the effects of the CFJ technology on the performance enhancement of the S809 airfoil and Phase VI wind turbine blade are explored in this study. Furthermore, the effects of the injection location and jet momentum coefficient are studied, and an adaptive jet momentum coefficient strategy of the CFJ technology is proposed. Results demonstrate that the CFJ technology can significantly improve the maximum lift coefficient and maximum corrected lift-to-drag ratio of the S809 airfoil. Moreover, the power coefficient of the Phase VI wind turbine blade at the low tip speed ratio is greatly enhanced as well. In particular, the maximum lift coefficient and maximum corrected lift-to-drag ratio of the typical S809 CFJ airfoil with adaptive $C_{\mu}$ are improved by $119.7 \%$ and $36.2 \%$, respectively. The maximum power coefficient of CFJ blade can be increased by $4.5 \%$, and the power coefficient of CFJ blade can be boosted by $226.7 \%$ when the tip speed ratio is 1.52 .
\end{abstract}

Keywords: co-flow jet; S809 airfoil; Phase VI; wind turbine blade; active flow control; performance enhancement

\section{Introduction}

As a kind of clean energy, wind energy has been paid more and more attention by various countries. According to preliminary wind power statistics published by the World Wind Energy Association (WWEA), the total capacity of all wind farms worldwide by the end of 2020 has reached 744 gigawatt, which is sufficient to generate $7 \%$ of the world's electricity demand [1]. As the basic elements of the wind turbine blade, the performance of the airfoils directly affects the aerodynamic characteristics of wind turbine blade, which are essential to the power generation of the wind turbine. Meanwhile, flow control technology has shown extremely significant advantages in improving the performance of airfoils and blades, and is one of the effective ways to greatly improve the performance of wind turbine airfoils and blades.

Many researchers have studied various passive and active flow control methods to enhance the performance of the wind turbine airfoil and blade, such as gurney flaps [2-6], vortex generators [7-11], synthetic jets [12-16], circulation controls [17,18], and plasma actuators [19-24]. Gamiz et al. [3] found that the power output of the five-megawatt wind turbine could be significantly increased by equipping with vortex generators (VGs) and Gurney flaps (GFs). Inigo et al. [6] implemented the GF to improve the aerodynamic performance of the DU91W(2)250 wind tunnel airfoil. Results showed that optimum GF lengths of the airfoil with maximum lift-to-drag ratios were between $0.25 \%$ and $0.75 \%$ 
chord length. Li et al. [10] carried out the wind tunnel tests and numerical simulations to explore the aerodynamic characteristics of the DU93-W-210 wind tunnel airfoil with VGs. It was found that the stall angle of attack and lift coefficient of the airfoil with VG were increased by $10^{\circ}$ and $48.7 \%$, respectively. Ye et al. [11] applied the VGs to the NREL wind turbine and demonstrated that the wind turbine with VGs achieved better aerodynamic performance compared to the baseline. Moshfeghi et al. [13] studied the effects of the synthetic jet actuators (SJAs) on the performance improvement of a wing composed of the NREL S809 airfoil by numerical methods. Results demonstrated that the stall and lift characteristics of the wing with SJA became worse when the flow was attacked, but the lift performance was enhanced when the flow was separated. Maldonado et al. [16] analyzed the power efficiency of the wind turbine with SJAs. Results indicated that the power demand of driving the wind turbine with SJAs could be reduced by $10.6 \%$ at $500 \mathrm{rpm}$. $\mathrm{Xu}$ et al. $[17,18]$ validated the effectiveness of the full circulation control (FCC) and partial circulation control (PCC) on the performance enhancement of wind turbine airfoils with the blunt trailing edge. Aono et al. [19] explored the effects of the plasma technology on the aerodynamic performance of the NREL S825 airfoil. Results showed that the shear layer separation near the leading edge was prevented by the direct momentum addition produced by the plasma actuator during the case setup. Li et al. [21,22] proved that the dynamic stall of the wind turbine airfoil could be effectively controlled by the plasma actuators. Aono et al. [23] used the dielectric barrier discharge plasma actuator to control the separated flow of the wind turbine and demonstrated that the leading edge separation could be effectively suppressed at high angle of attack. Zhu et al. [25] presented a novel flow control strategy which could reduce the power consumptions of the blowing, synthetic, and plasma jet actuators, resulting in the performance enhancement of the vertical axis wind turbine.

Moreover, a novel active flow control technology, named co-flow jet (CFJ), was proposed and developed by Zha et al. [26-36]. The CFJ airfoil, which has an injection slot near the leading edge and a suction slot near the trailing edge on the airfoil suction surface, has shown the significant lift enhancement and drag reduction with low power expenditure [26-28]. Because the mass flux of the injection is equal to that of the suction, the zero net mass flux can be achieved. Meanwhile, Yang et al. [29-32] numerically demonstrated that the CFJ airfoil and cylinder could achieve the super-lift coefficient that far exceeded the theoretical limit, which was proved by Zha et al. [33] for the first time in experiment. To apply embedded micro-compressor actuator to the CFJ airfoil during the tunnel experiment, Ren et al. [34-36] designed the injection and suction duct of the CFJ airfoil, and simulated the 3D CFJ airfoil with an embedded micro-compressor actuator. Zhang et al. [37] explored the numerical and experimental methods to achieve CFJ technology with fans inside the duct. Results demonstrated that the maximum lift coefficient of the CFJ airfoil could be increased by at least $50 \%$ and the drag coefficient could be negative at small angles of attack. Therefore, the CFJ technology is one of the most effective means to greatly improve the performance of the wind turbine airfoil, thereby improving the performance of the wind turbine.

The CFJ technology has been applied to the propeller or rotor airfoil. Zhu et al. [38] applied the CFJ technology to the near space propeller, whose efficiency was increased by more than 5\%. Yang et al. [39] analyzed the dynamic stall characteristics of the CFJ rotor airfoils and demonstrated that the CFJ technology could remove the dynamic stall phenomenon occurring on rotor airfoils. The parametric studies of the CFJ technology applied to the various conventional airfoils have been implemented by researchers. Zha et al. [40] and Wang et al. [41] explored the effect of the injection slot size on the CFJ airfoils based on the NACA0025 airfoil. Results demonstrated that the stall and lift characteristics of CFJ airfoils with the small injection slot size could be greatly enhanced, whereas the drag characteristics of CFJ airfoils with the large injection slot size could be effectively improved. Lefebvre et al. [42,43] implemented the CFJ airfoil trade study based on the NACA23121 airfoil when the angle of attack (AoA) was $10^{\circ}$ and the injection location was found to have 
significant influence on the energy consumption and aerodynamic efficiency. Xu et al. [44] studied the effects of the key parameters, including jet momentum coefficient, injection slot size, and injection location, on the aerodynamic performance of the co-flow jet airfoil based on the NACA6415 airfoil at the low-Reynolds-number condition and the different angles of attack. The results showed that the injection location and suction location had little effects on the energy consumption and aerodynamic efficiency, and the conclusion about the effect of the injection location on the CFJ airfoil was very different from that of Lefebvre et al. $[42,43]$. The differences may be caused by the various CFJ airfoils and computational conditions.

Moreover, the CFJ technology has been applied to the wind turbine airfoil and blade. The static aerodynamic performance and dynamic stall control of the NREL S809 airfoil using the CFJ technology were numerically investigated by $\mathrm{Xu}$ et al. $[45,46]$. The results showed that the CFJ technology was proved to have the positive effect in increasing lift, reducing drag, and suppressing dynamic stall of the S809 airfoil. However, the influence of the energy consuming, which was only researched individually, was not included in the lift-to-drag ratio and only one CFJ airfoil configuration was studied. Sun et al. [47] numerically investigated the aerodynamic performance of the vertical axis wind turbine using the CFJ technology and demonstrated that the CFJ technology could recover some of the power loss due to blade stall. When the jet momentum coefficient was bigger, the control effect was better. In addition, $\mathrm{Xu}$ et al. [48] implemented the investigation of co-flow jet active flow control for wind turbine airfoil. The effects of injection slot size, injection slot location, suction slot size, suction slot location, and injection total pressure were studied. The optimum configuration was able to increase the maximum lift coefficient by $42.3 \%$. But the value ranges of the parameters were not wide enough and only three values were taken for each parameter. Moreover, the corrected lift-to-drag ratio of the optimum configuration had no increase when compared with the baseline. Meanwhile, $\mathrm{Xu}$ et al. [49] studied the performance enhancement of the Phase VI wind turbine and demonstrated that the power output of the CFJ blade could be increased by $207.7 \%$ at the jet momentum coefficient of 0.23 . Although the power output increment of the CFJ wind turbine was large, the required jet momentum coefficient was too large, which led to difficulties in applications. Therefore, it is meaningful to develop a novel CFJ strategy that can achieve a large performance enhancement of the wind turbine at a small jet momentum coefficient.

In order to further explore the performance improvement effects of CFJ technology on the wind turbine airfoil, it is necessary to further carry out research to obtain CFJ technology strategies that can simultaneously improve the maximum lift coefficient and corrected lift-to-drag ratio of wind turbine airfoil. Based on the accumulated research experiences, the injection location and jet momentum coefficient of CFJ technology have significant influence on the performance improvement of S809 airfoil. Therefore, under the condition that other parameters of CFJ technology remain unchanged, the effects of the injection location and jet momentum coefficient on the performance of S809 airfoil are explored in the wider value ranges using the CFD method. The optimum strategy of CFJ technology for improving the performance of the S809 airfoil is proposed. Moreover, the performance enhancement of the Phase VI wind turbine blade using the CFJ technology is also explored. The research results have significant reference value for the performance improvement of wind turbines.

\section{Methodology}

\subsection{CFJ Airfoil Configuration Design}

The S809 airfoil designed by the National Renewable Energy Laboratory (NREL) for the horizontal axis wind turbine blade is selected as the baseline airfoil. The NREL S809 airfoil has the maximum thickness of $21 \%$ and more detailed parameters can be found in [50]. 
With reference to literature documentation $[29,45,46]$, the NREL S809 CFJ airfoil is designed, as sketched in Figure 1a. The airfoil suction surface between the injection slot near the leading edge and suction slot near the trailing edge are translated downward to create these slots, and the translation distance is defined as the suction surface translation (SST). To achieve different size of the injection slot and suction slot, the portions of the airfoil suction surface near the injection slot and suction slot are reconstructed. The ducts near the injection slot and suction slot are included in the computational model of the S809 CFJ airfoil to ensure the injection slot and suction slot possessing more realistic velocity profiles, as sketched in Figure 1b. A small amount of mass flow is pressurized by the pump inside the airfoil and injected into the main flow through the injection slot. Meanwhile, the same amount of mass flow is sucked in through the suction slot.

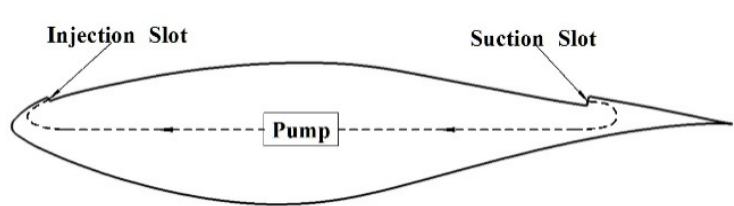

(a)

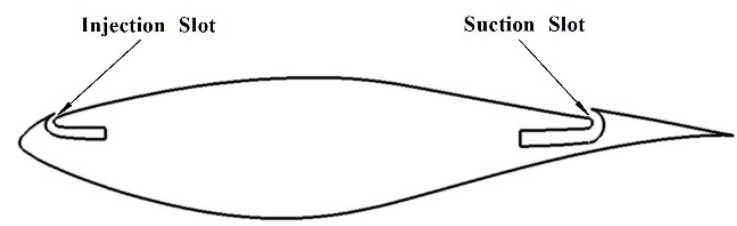

(b)

Figure 1. (a) Schematic diagram of the S809 CFJ airfoil; (b) computational model of the S809 CFJ airfoil.

\subsection{Numerical Methods}

The Reynolds-Averaged Navier-Stokes (RANS) equations with the $k-\omega$ SST twoequation turbulence model are used in this research. The finite-volume method with second order spatial accuracy is utilized. HP MPI parallel computing technique is implemented to save the simulation time.

Considering the sharp trailing edge of the $\$ 809$ airfoil, the C-type structured grid with the domain whose radius and length are 50 and 100 times of the chord length is adopted for the simulation, as shown in Figure 2. The velocity inlet is set as the inflow condition at the inlet boundary, and the pressure outlet is set as the outlet condition at the exit boundary. The massflow inlet is set as the injection condition, where the mass flow rate is specified. Meanwhile, the pressure outlet is set as the suction condition, where the pressure change is iterated to achieve the specified amount of mass flow. The no-slip wall boundaries are set at the solid surfaces of the airfoil and the duct.

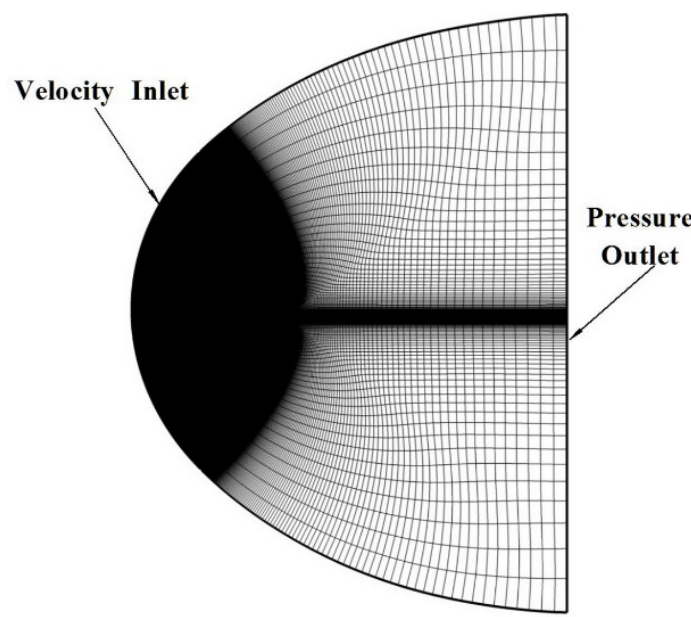

(a)

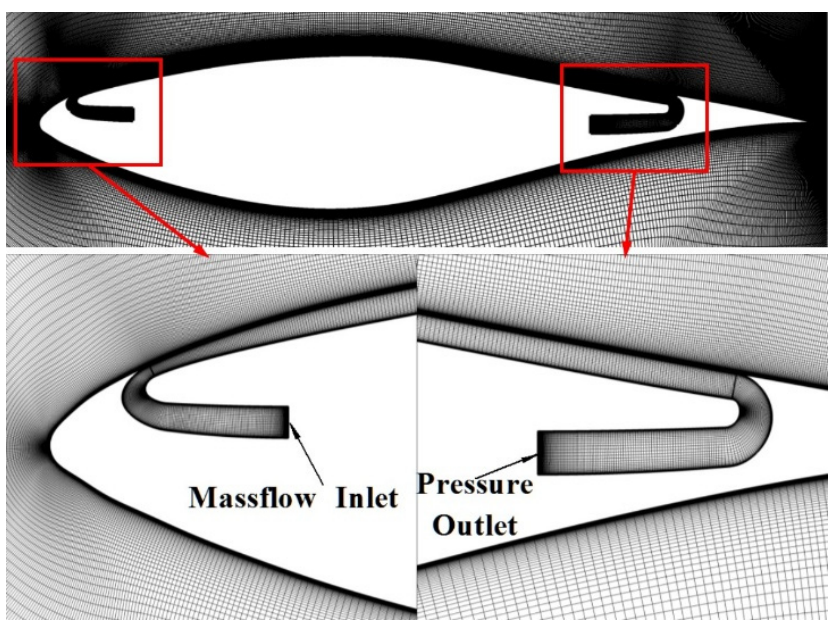

(b)

Figure 2. Computational grid and boundary conditions of the airfoil. (a) Overall grid; (b) close-up view of the S809 CFJ airfoil grid. 


\subsection{CFJ Airfoil Parameters}

The jet intensity of the CFJ airfoil is evaluated using the jet momentum coefficient $C_{\mu}$, which is defined as:

$$
C_{\mu}=\frac{\dot{m} V_{j}}{0.5 \rho_{\infty} V_{\infty}^{2} S}
$$

where $\dot{m}$ is the injection mass flow rate, $V_{j}$ and $V_{\infty}$ are the injection velocity and free-stream velocity respectively, $\rho_{\infty}$ is the free-stream density, and $S$ is the reference area of the airfoil.

According to the reference [42,43], the power consumption and corresponding power coefficient required by the pump inside the $\mathrm{CFJ}$ airfoil are defined as:

$$
\begin{gathered}
P=\frac{\dot{m} C_{p} T_{0}}{\eta}\left[\left(\frac{p_{01}}{p_{02}}\right)^{\frac{\gamma-1}{\gamma}}-1\right] \\
P_{C}=\frac{P}{0.5 \rho_{\infty} V_{\infty}^{3} S}
\end{gathered}
$$

where $C_{p}$ denotes the specific heat capacity at the constant pressure, $T_{0}$ indicates the total temperature, $P_{01}$ and $P_{02}$ imply the total pressure at the pump exit and inlet respectively, and $\gamma$ is the specific heat ratio, which is equal to 1.4 for air. $\eta$ is the pump efficiency, which is taken to be 1.0 in this study.

The conventional lift-to-drag ratio is defined as:

$$
L / D=C_{L} / C_{D}
$$

where $C_{L}$ and $C_{D}$ are respectively the lift coefficient and drag coefficient considering the jet reactionary force for the $\mathrm{CFJ}$ airfoil.

To take into account the influence of the power consumption on the aerodynamic performance of the CFJ airfoil, the corrected lift-to-drag ratio including the pump power is defined as [42,43]:

$$
(L / D)_{e}=\frac{L}{D+P / V_{\infty}}=\frac{C_{L}}{C_{D}+P_{C}}
$$

\section{Results and Discussions}

\subsection{Baseline Simulation and Validation}

The performance of 5809 airfoil has been conducted in the low-turbulence wind tunnel of the Delft University of Technology Low Speed Laboratory at the free transition and fixed transition conditions, respectively [50]. As no transition model is used in the simulations, the experimental results at fixed transition condition by roughness at $0.02 \mathrm{c}$ on the upper surface and $0.05 \mathrm{c}$ at lower surface are selected to validate the numerical methods. Based on the experimental conditions, the Mach number 0.15 and Reynolds number $2.0 \times 10^{6}$ are adopted for all cases in this study. The grid refinement study is carried out with three different grid resolutions, including a coarse grid of $1001 \times 102$, a medium grid of $1501 \times 144$ and a fine grid of $2001 \times 176$. The detailed parameters of the three grids are tabulated in Table 1.

Figure 3 shows the aerodynamic coefficients comparisons of the experimental and numerical results with various grid resolutions. The numerical lift and drag coefficients agree well with the experimental results when the AoA is less than $10^{\circ}$. However, the numerical lift coefficients are slightly higher than experimental results when the AoA is above $10^{\circ}$. This discrepancy may be caused by the fact that all RANS models cannot predict the turbulence stress levels accurately in large separation zones. 
Table 1. Detailed parameters of the S809 airfoil grids (airfoil chord length $=600 \mathrm{~mm}$ ).

\begin{tabular}{cccc}
\hline Parameters & Coarse Grid & Medium Grid & Fine Grid \\
\hline Wrap-around points & 801 & 1101 & 1401 \\
Normal layers & 102 & 144 & 176 \\
Wake points & 101 & 201 & 301 \\
Leading edge spacing/mm & 0.12 & 0.06 & 0.03 \\
Trailing edge spacing/mm & 0.48 & 0.24 & 0.12 \\
First layer spacing/mm & 0.006 & 0.006 & 0.006 \\
Growth rate & 1.15 & 1.10 & 1.08 \\
Total number of nodes & 102,102 & 216,144 & 352,176 \\
\hline
\end{tabular}

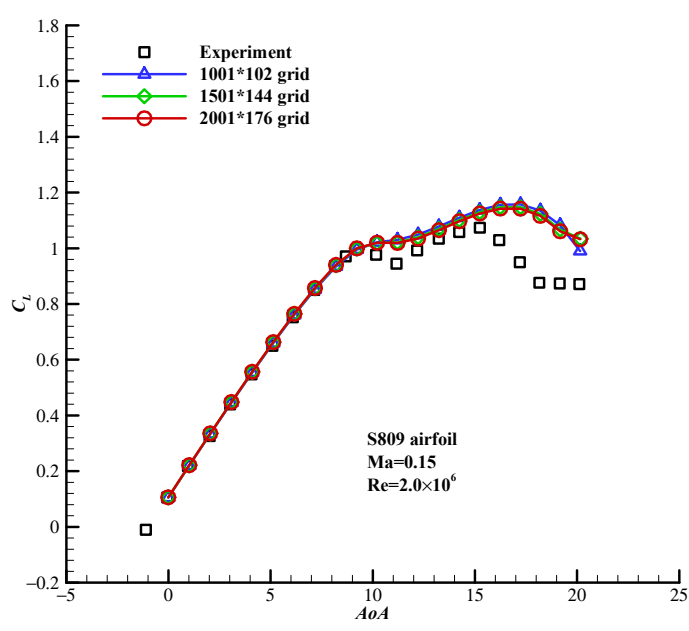

(a)

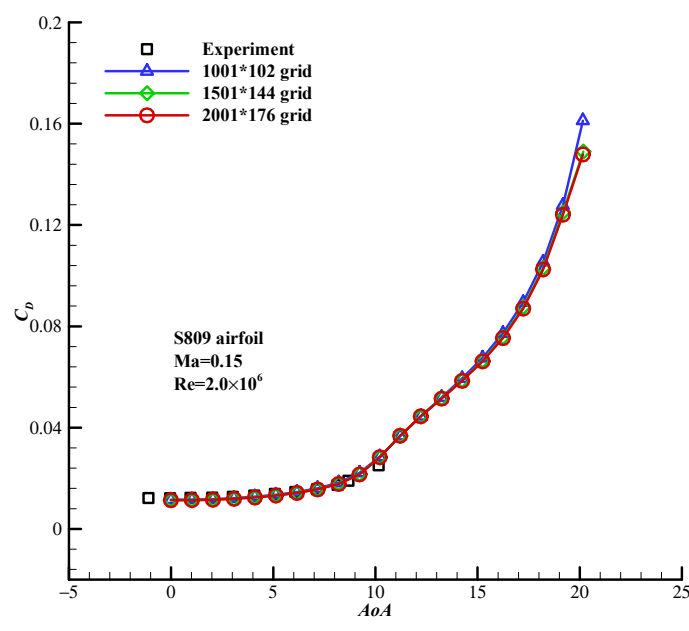

(b)

Figure 3. Aerodynamic coefficients comparisons of the experimental and numerical results with various grid resolutions at $\mathrm{Ma}=0.15, \operatorname{Re}=2.0 \times 10^{6}$. (a) Lift coefficient; (b) drag coefficient.

The comparisons of aerodynamic coefficients with different grid resolutions at three typical AoAs are given in Table 2. When AoA is $0^{\circ}$, the finer the grid, the closer the lift coefficient is to the experimental result while the drag coefficient is the opposite. But the error of drag coefficients using the coarse and medium grid is merely $1.1 \%$. Furthermore, for the drag coefficients and lift coefficients, the differences between the medium and fine grid are very small. The same conclusions about the lift coefficients can be drawn when AoA is $8.2^{\circ}$ and $12.22^{\circ}$. But the finer the grid, the closer the drag coefficient is to the experimental result when AoA is $8.2^{\circ}$. There is no experimental data of drag coefficient when AoA is $12.22^{\circ}$ and the drag coefficients using the three grids are close to each other. Thus, by compromising between the computational complexity and accuracy, the medium grid of $1501 \times 144$ is used in this paper.

Table 2. S809 airfoil aerodynamic coefficients comparisons between simulations and experiments $\left(\mathrm{Ma}=0.15, \operatorname{Re}=2.0 \times 10^{6}\right)$.

\begin{tabular}{cccccc}
\hline AoA & $\begin{array}{c}\text { Aerodynamic } \\
\text { Coefficients }\end{array}$ & Experiment & Coarse Grid & Medium Grid & Fine Grid \\
\hline \multirow{2}{*}{$0.00^{\circ}$} & $C_{L}$ & 0.11092 & 0.10619 & 0.10700 & 0.10725 \\
& $C_{D}$ & 0.01216 & 0.01152 & 0.01139 & 0.01134 \\
\multirow{2}{*}{$8.20^{\circ}$} & $C_{L}$ & 0.94007 & 0.93473 & 0.93897 & 0.94038 \\
& $C_{D}$ & 0.01755 & 0.01825 & 0.01785 & 0.01775 \\
\multirow{2}{*}{$12.22^{\circ}$} & $C_{L}$ & 0.99410 & 1.04870 & 1.03923 & 1.03477 \\
& $C_{D}$ & - & 0.04451 & 0.04442 & 0.04448 \\
\hline
\end{tabular}




\subsection{Effect of the Injection Location}

The injection slot size, suction slot size and suction surface translation are fixed to $0.65 \%$ chord, $1.33 \%$ chord and $1.43 \%$ chord, respectively. The suction location is fixed to the $80 \%$ chord-wise location from the leading edge and the $C_{\mu}$ is fixed to 0.04 . The injection location varies from a $1 \%$ chord-wise location to a $20 \%$ chord-wise location from the leading edge. Effects of six injection locations with the 1\%, $\%, 9 \%, 13 \%, 17 \%$, and $20 \%$ chord-wise locations, as illustrated in Figure 4, on the performance of S809 CFJ airfoil are investigated.

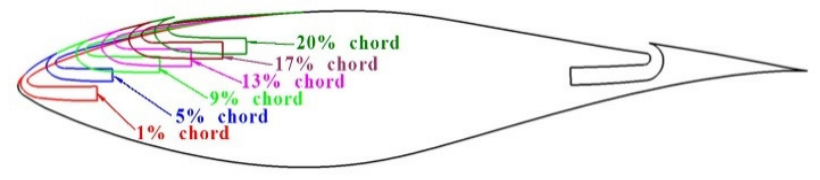

Figure 4. S809 CFJ airfoils with various injection locations.

Figure 5 presents aerodynamic coefficients comparisons of S809 CFJ airfoils with various injection locations. When the AoA is below $8.2^{\circ}$, the lift and drag coefficients of S809 CFJ airfoils with various injection locations are almost the same. As the injection slot is moved downstream, the power consumption coefficient decreased when the AoA is below $2.02^{\circ}$ and increased when the AoA is between $2.02^{\circ}$ and $8.2^{\circ}$. Thus, when the AoA is below $8.2^{\circ}$, the corrected lift-to-drag ratio of S809 CFJ airfoil increased as the injection slot is moved upstream. But at the large AoA, the more downstream the injection location, the better the lift characteristic of S809 CFJ airfoil, resulting in the better corrected liftto-drag ratio of S809 CFJ airfoil. The lift curves of S809 CFJ airfoils with the $17 \%$ and $20 \%$ chord-wise injection locations almost coincide expect that the AoA is above $16.24^{\circ}$. The curvature of the suction surface of the $\$ 809$ airfoil at the 1\% chord-wise location is larger than those at other chord-wise locations. The adverse pressure gradient region of the suction surface is near 1\% chord-wise location at high angle of attack. When the injection slot of S809 CFJ airfoil is located at 1\% chord-wise location, more energies are needed to overcome the adverse pressure gradient. Meanwhile, the existence of the injection destroys the smoothness of the suction surface of the $\$ 809$ airfoil, which will cause the separation point to move upstream and the separation region to become larger when stall occurs. The more upstream the injection, the greater the fluence on the smoothness of the suction surface. Therefore, when the S809 CFJ airfoil with the 1\% chord-wise injection location stalls at the AoA of $8.2^{\circ}$, the jet momentum coefficient of 0.04 is not enough to overcome the large adverse pressure gradient and the drag is suddenly increased. Table 3 presents the maximum lift coefficients and maximum lift-to-drag ratios of $\mathrm{S} 809 \mathrm{CFJ}$ airfoils with various injection locations. As the injection slot is moved downstream, the maximum lift coefficient is increased. The range of corrected lift-to-drag ratio increment of S809 CFJ airfoil with various injection locations is between $29.3 \%$ and $36.2 \%$ when compared with the baseline. When the injection slot is at the $1 \%$ chord-wise location, the maximum lift coefficient and maximum lift-to-drag ratio of S809 CFJ airfoil are 2.6\% lower and 34.3\% higher than that of the baseline, respectively. The S809 CFJ airfoil with the 9\% chord-wise injection location has the optimum maximum corrected lift-to-drag ratio, which is $36.2 \%$ higher than that of the baseline. The S809 CFJ airfoil with the $20 \%$ chord-wise injection location has the optimum maximum lift coefficient, which is $76.5 \%$ higher than that of the baseline.

Figures 6 and 7 show the Mach number contours and streamlines of S809 CFJ airfoils with various injection locations when the AoAs are $8.2^{\circ}$ and $16.24^{\circ}$, respectively. It can be seen that at $8.2^{\circ} \mathrm{AoA}$, the flows of S809 CFJ airfoils with various injection locations are attached. The high local Mach number region of S809 CFJ airfoil is near the leading edge. Thus, the closer the injection location is to the leading edge, the easier it is for the mass flow to be injected into the main flow and the less the energy is consumed. This is why the S809 $\mathrm{CFJ}$ airfoil with the injection location near the leading edge has low power consumption coefficient and high corrected lift-to-drag ratio at the small AoA. At $16.24^{\circ} \mathrm{AoA}$, the large flow separations appear on the suction surfaces of S809 CFJ airfoils with the $1 \%, 5 \%$, 
and 9\% chord-wise injection locations, but the flows of S809 CFJ airfoils with the 13\%, 17\%, and $20 \%$ chord-wise injection locations are attached. The separation region is large and the separation point is near the leading edge at $16.24^{\circ}$ AoA. When the injection location is close to the leading edge, the injection location is too close to the adverse pressure gradient region and the jet energy of $\mathrm{S} 809 \mathrm{CFJ}$ airfoil with $0.04 C_{\mu}$ is not enough to suppress the flow separation, and most of the jet energy is converted into heat. When the injection slot is moved downstream, the injection location is near the vortex core and it is easier to inject the jet energy into the main flow to suppress the large flow separation.

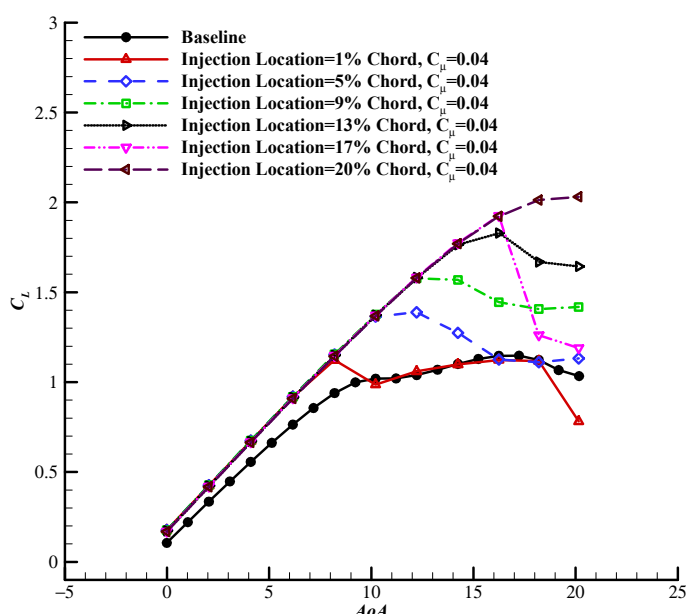

(a)

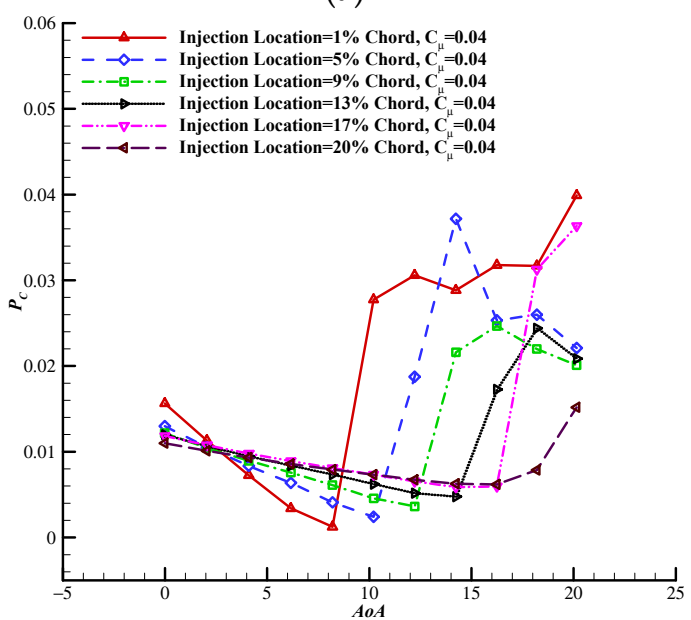

(c)

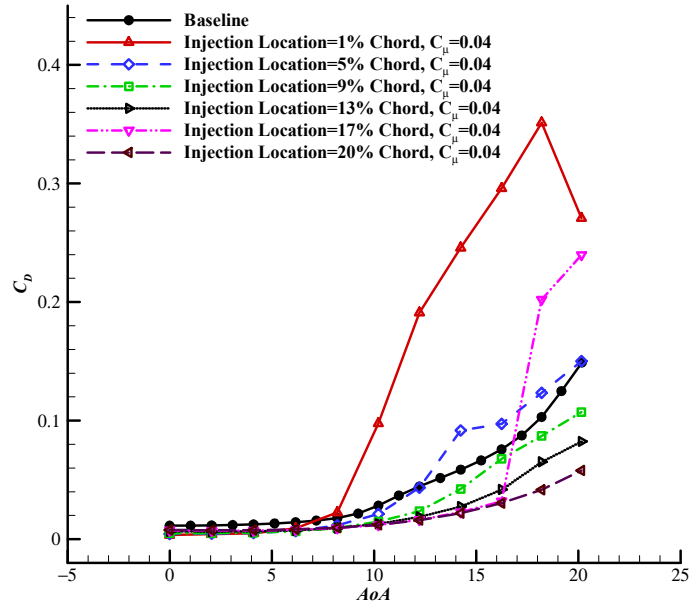

(b)

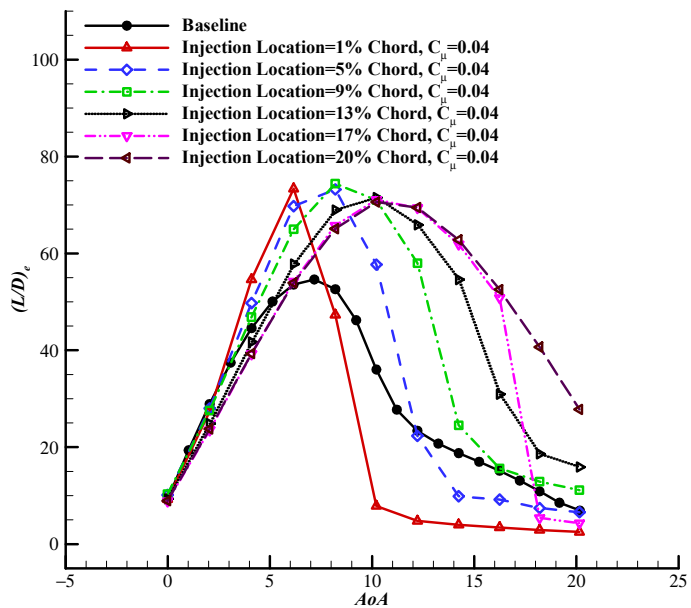

(d)

Figure 5. Aerodynamic coefficients comparisons of the S809 CFJ airfoils with various injection locations $\left(C_{\mu}=0.04\right)$. (a) Lift coefficient; (b) drag coefficient; (c) power coefficient; (d) corrected lift-to-drag ratio.

Table 3. Maximum lift coefficients and maximum lift-to-drag ratios of S809 CFJ airfoils with various injection locations $\left(C_{\mu}=0.04\right)$.

\begin{tabular}{ccccc}
\hline Injection Location & $\boldsymbol{C}_{\text {Lmax }}$ & $\Delta \boldsymbol{C}_{\text {Lmax }}$ & $(\boldsymbol{L} / \mathbf{D})_{\text {emax }}$ & $\Delta(\boldsymbol{L} / \mathbf{D})_{\text {emax }}$ \\
\hline baseline & 1.15 & - & 54.61 & - \\
$1 \%$ chord-wise location & 1.12 & $-2.6 \%$ & 73.34 & $34.3 \%$ \\
$5 \%$ chord-wise location & 1.39 & $20.9 \%$ & 73.20 & $34.0 \%$ \\
$9 \%$ chord-wise location & 1.58 & $37.4 \%$ & 74.40 & $36.2 \%$ \\
$13 \%$ chord-wise location & 1.83 & $59.1 \%$ & 71.52 & $31.0 \%$ \\
$17 \%$ chord-wise location & 1.93 & $67.8 \%$ & 71.08 & $30.2 \%$ \\
$20 \%$ chord-wise location & 2.03 & $76.5 \%$ & 70.59 & $29.3 \%$ \\
\hline
\end{tabular}




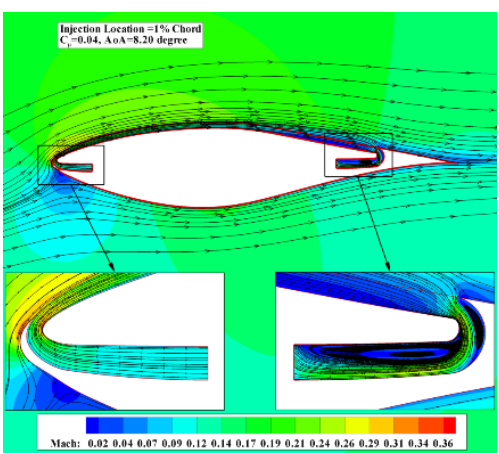

(a)

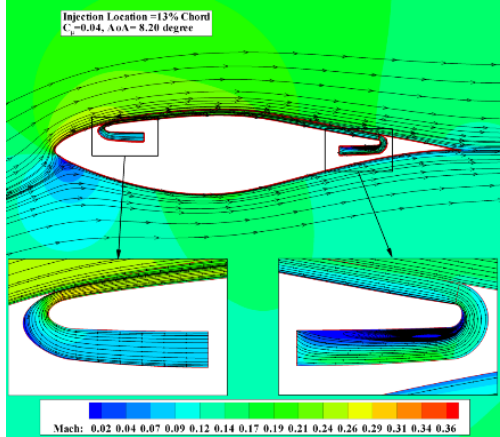

(d)

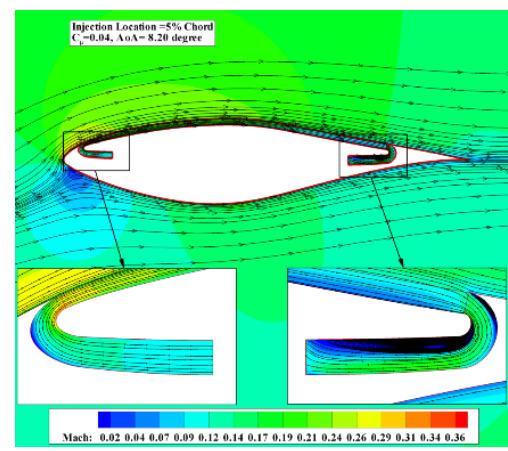

(b)

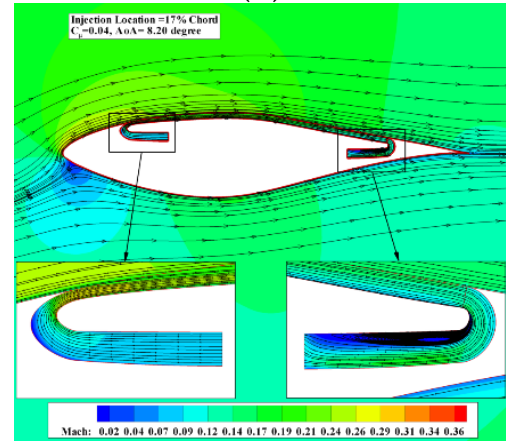

(e)

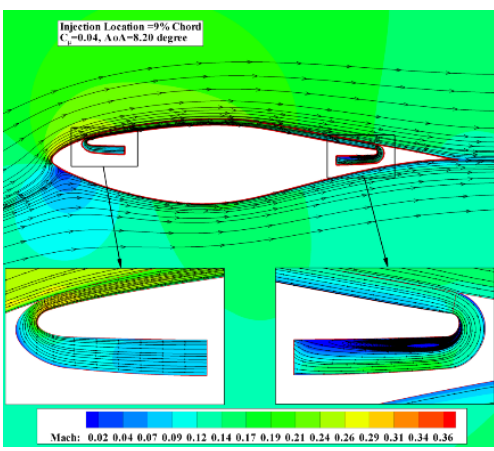

(c)

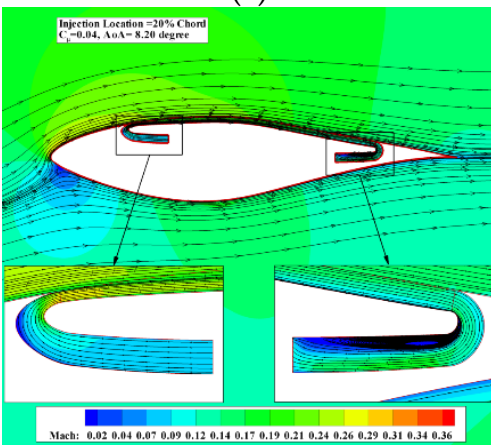

(f)

Figure 6. Mach number contours and streamlines of S809 CFJ airfoils with various injection locations $\left(C_{\mu}=0.04\right.$ and AoA $\left.=8.2^{\circ}\right)$. (a) Injection location $=1 \%$ chord; (b) injection location $=5 \%$ chord; $(\mathbf{c})$ injection location $=9 \%$ chord; $(\mathbf{d})$ injection location $=13 \%$ chord; $(\mathbf{e})$ injection location $=17 \%$ chord $;(\mathbf{f})$ injection location $=20 \%$ chord.

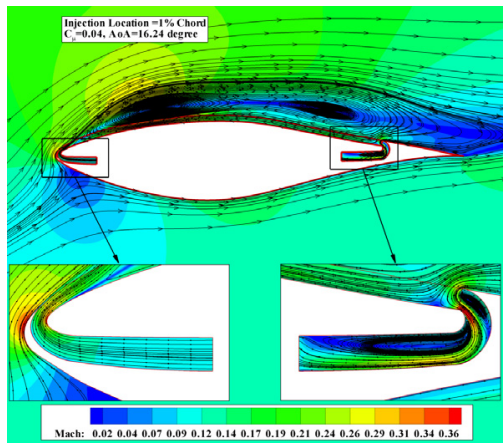

(a)

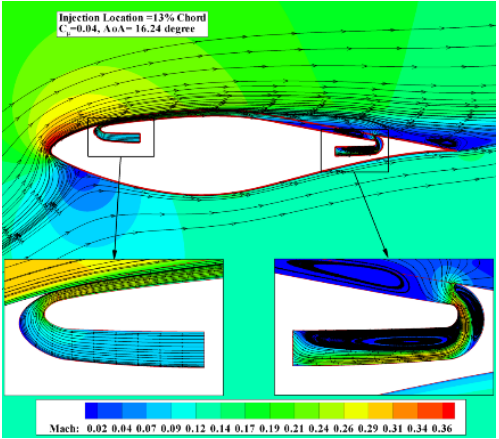

(d)

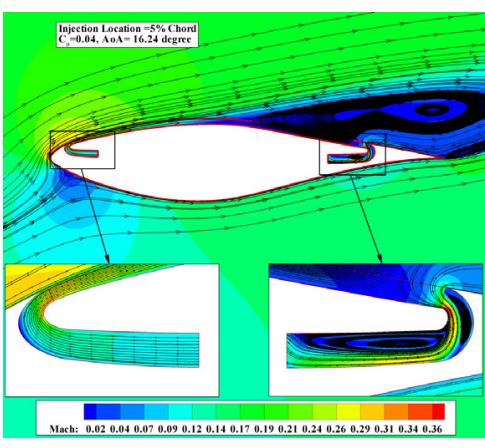

(b)

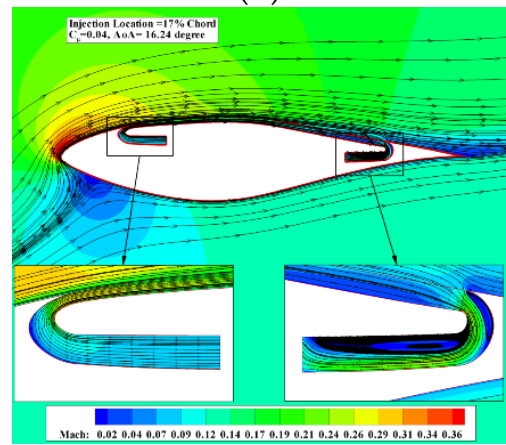

(e)

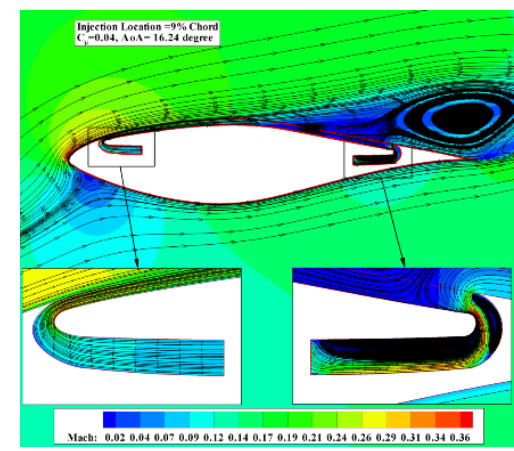

(c)

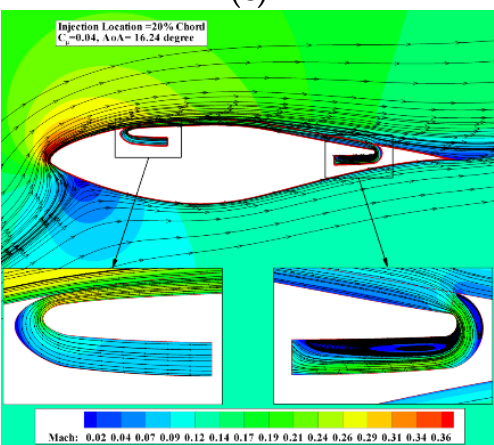

(f)

Figure 7. Mach number contours and streamlines of S809 CFJ airfoils with various injection locations $\left(C_{\mu}=0.04\right.$ and $\mathrm{AoA}=16.24^{\circ}$ ). (a) Injection location $=1 \%$ chord; (b) injection location $=5 \%$ chord; (c) injection location $=9 \%$ chord; (d) injection location $=13 \%$ chord; (e) injection location $=17 \%$ chord; (f) injection location $=20 \%$ chord. 
In order to more intuitively characterize the performance enhancement of the CFJ technology on the S809 airfoil, the surface pressure coefficient distributions comparisons and velocity profiles comparisons of the baseline and S809 CFJ airfoil are implemented, as shown in Figures 8 and 9. It is obvious to find that CFJ technology can greatly increase the peak of negative pressure at the leading edge of the S809 airfoil, and the negative pressure region is expanded especially when the AoA is $16.24^{\circ}$. Therefore, the lift coefficient of the S809 airfoil can be significantly enhanced by the CFJ technology. In Figure 9, the variable of $x$-axis is the dimensionless velocity near the suction surface of the airfoils, and the variable of $y$-axis is the distance from the suction surface given in the percent chord length. The velocity profiles of the baseline and S809 CFJ airfoil at the 50\% and 70\% chord-wise locations when the AoAs are $8.2^{\circ}$ and 16.24 are taken perpendicular to suction surface of the airfoil. It can be seen that when AoA is $8.2^{\circ}$, no separation occurs in the boundary layers of the baseline and S809 CFJ airfoil, but the velocity profiles of S809 CFJ airfoil are fuller than those of the baseline. When AoA is $16.24^{\circ}$, large separations occur in the boundary layer of the baseline, while the flow in the boundary layer of the S809 CFJ airfoil is still attached because the jet energy is injected into the main flow.

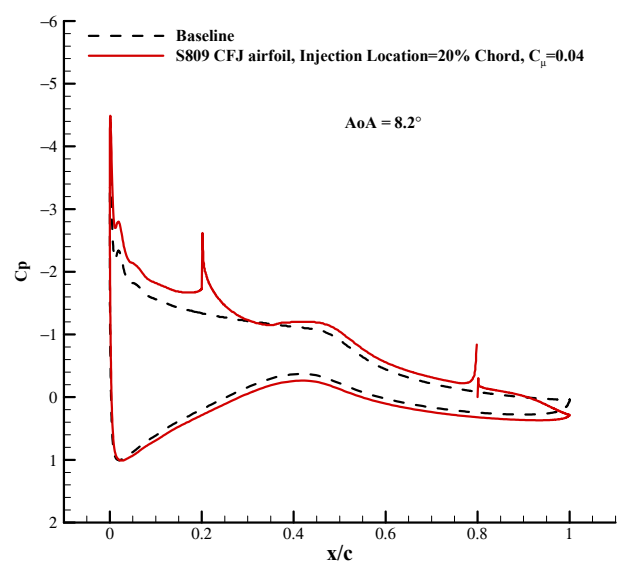

(a)

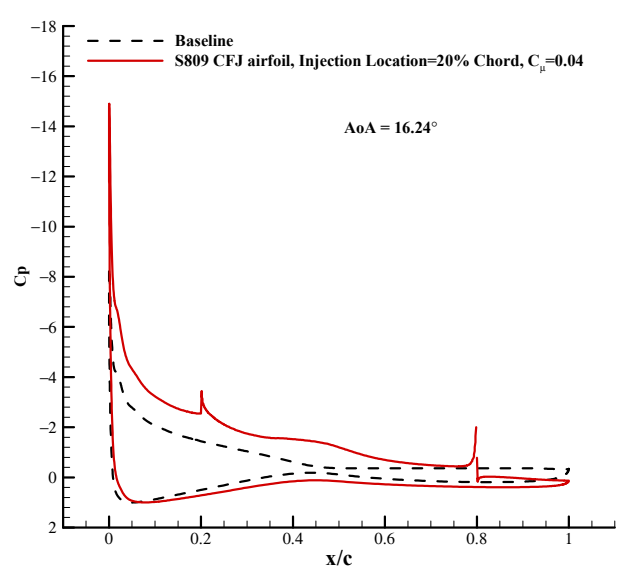

(b)

Figure 8. Comparisons of surface pressure coefficient distributions of the baseline and S809 CFJ airfoil with 20\% chord-wise injection location $\left(C_{\mu}=0.04\right)$. (a) $\mathrm{AoA}=8.2^{\circ} ;(\mathbf{b}) \mathrm{AoA}=16.24^{\circ}$.

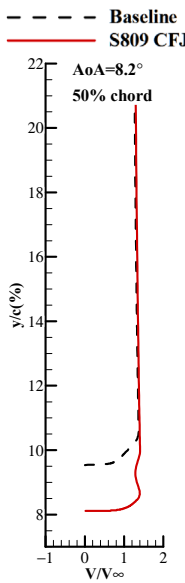

(a)

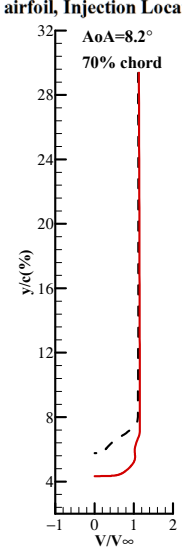

(b)

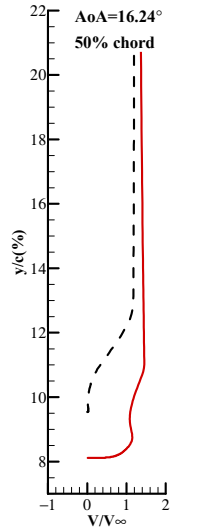

(c)

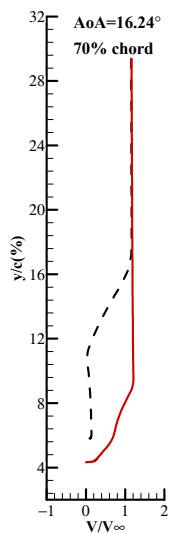

(d)

Figure 9. Comparisons of velocity profiles near the suction surfaces of the baseline and S809 CFJ airfoil with $20 \%$ chord-wise injection location $\left(C_{\mu}=0.04\right)$. (a) $\mathrm{AoA}=8.2^{\circ}, 50 \%$ chord-wise location; (b) $\mathrm{AoA}=8.2^{\circ}, 70 \%$ chord-wise location; (c) $\mathrm{AoA}=16.24^{\circ}, 50 \%$ chord-wise location; (d) AoA $=16.24^{\circ}$, $70 \%$ chord-wise location. 
The unsteady simulations for the baseline and S809 CFJ airfoil with $13 \%$ chord-wise injection location are implemented to explore the effects of CFJ technology on the vortex structures when AoA is $20.15^{\circ}$. The time histories of lift coefficient and drag coefficient of the baseline and S809 CFJ airfoil are given in Figures 10 and 11, respectively. It can be found that the amplitudes of lift coefficient and drag coefficient of S809 CFJ airfoil are significantly smaller than those of the baseline. The lift coefficient amplitude of S809 CFJ airfoil is only 0.004 while that of the baseline is 0.0225 . The drag coefficient amplitude of S809 CFJ airfoil is only 0.0016 while that of the baseline is 0.0076 . Moreover, the vortex shedding frequency of S809 airfoil, which is $139 \mathrm{~Hz}$, is larger than that of the baseline, which is $73 \mathrm{~Hz}$. Figure 12 shows the instantaneous Mach number contours and streamlines of the baseline and S809 CFJ airfoil within one time cycle. The separation region of S809 CFJ airfoil is obviously smaller than that of the baseline. Meanwhile, the secondary vortex of S809 CFJ airfoil near the trailing edge, induced by the primary vortex, is also smaller than that of the baseline.

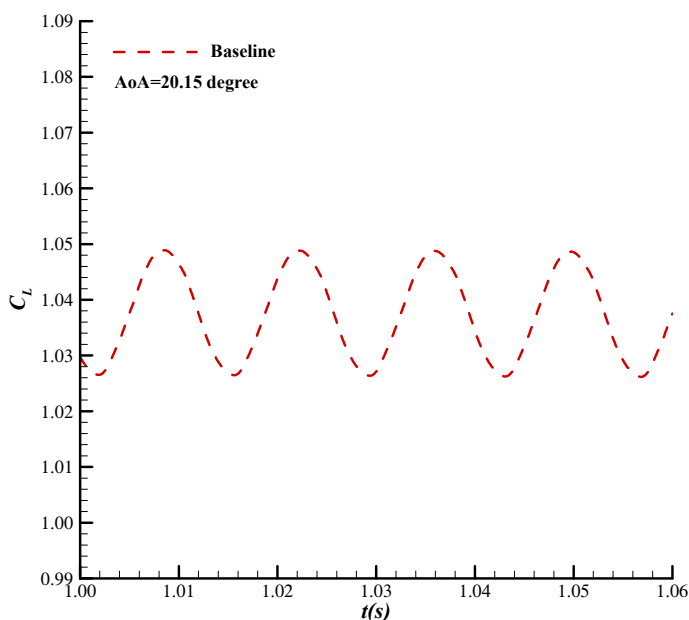

(a)

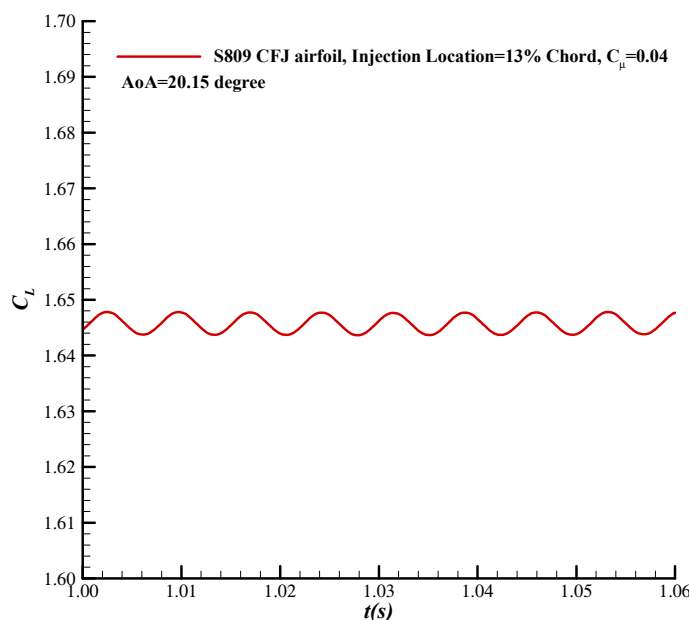

(b)

Figure 10. Time histories of lift coefficient of the baseline and S809 CFJ airfoil with $13 \%$ chord-wise injection location $\left(C_{\mu}=0.04\right.$ and $\left.\mathrm{AoA}=20.15^{\circ}\right)$. (a) Baseline; (b) S809 CFJ airfoil.

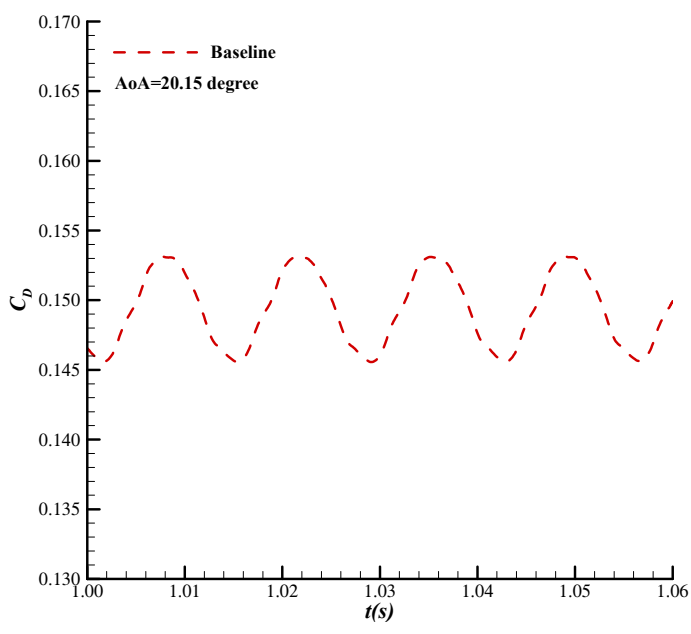

(a)

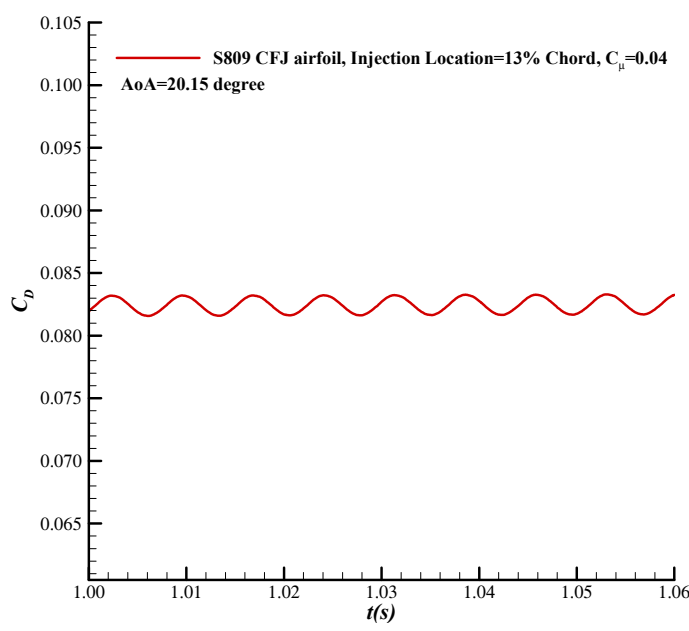

(b)

Figure 11. Time histories of drag coefficient of the baseline and S809 CFJ airfoil with 13\% chord-wise injection location $\left(C_{\mu}=0.04\right.$ and $\left.\mathrm{AoA}=20.15^{\circ}\right)$. (a) Baseline; (b) S809 CFJ airfoil. 


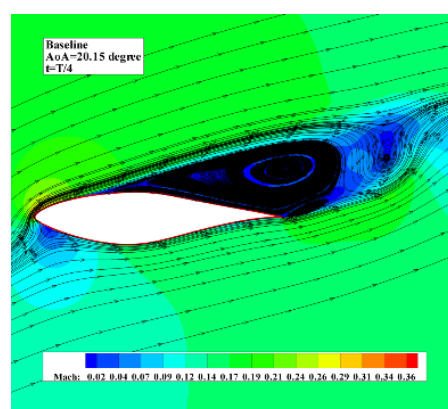

(a)

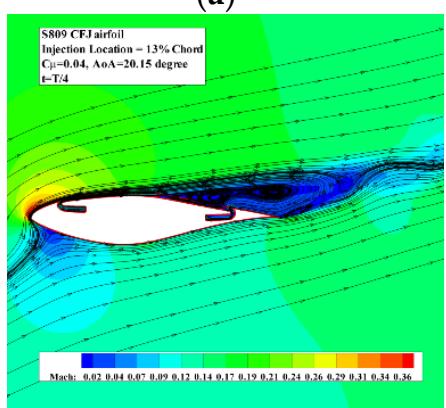

(e)

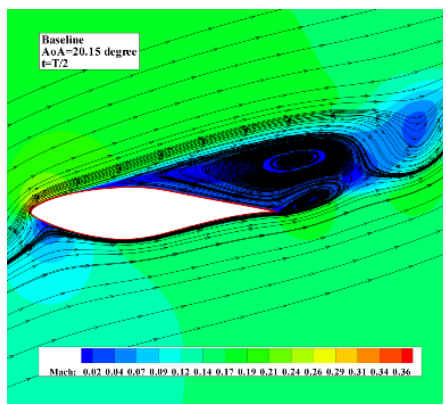

(b)

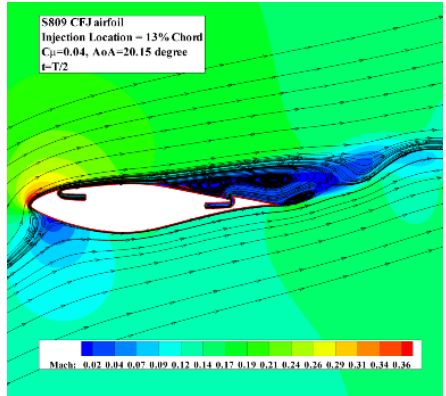

(f)

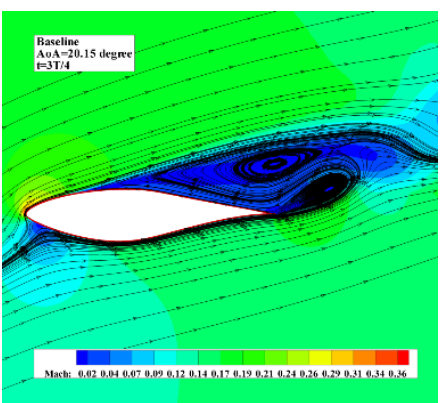

(c)

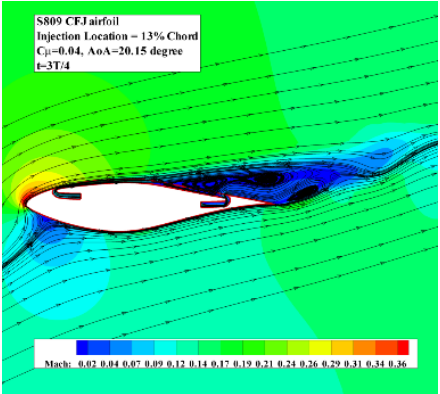

(g)

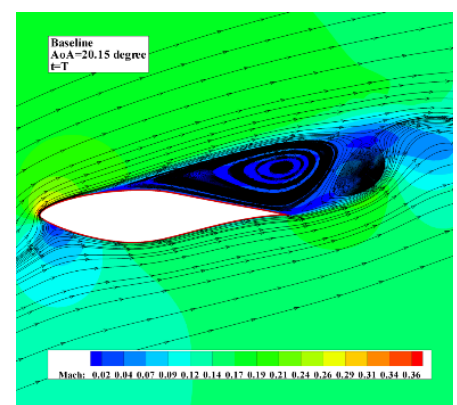

(d)

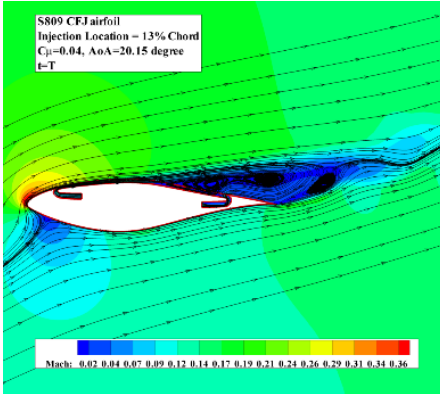

(h)

Figure 12. Instantaneous Mach number contours and streamlines of the baseline and S809 CFJ airfoil with $13 \%$ chord-wise injection location $\left(C_{\mu}=0.04\right.$ and AoA $\left.=20.15^{\circ}\right)$. (a) Baseline, $\mathrm{t}=\mathrm{T} / 4 ;(\mathbf{b})$ baseline, $\mathrm{t}=\mathrm{T} / 2 ;(\mathbf{c})$ baseline, $\mathrm{t}=3 \mathrm{~T} / 4 ;(\mathrm{d})$ baseline, $\mathrm{t}=\mathrm{T}$; (e) S809 CFJ airfoil, $\mathrm{t}=\mathrm{T} / 4$; (f) S809 CFJ airfoil, $\mathrm{t}=\mathrm{T} / 2$; (g) S809 CFJ airfoil, $\mathrm{t}=3 \mathrm{~T} / 4$; (h) S809 CFJ airfoil, $\mathrm{t}=\mathrm{T}$.

\subsection{Effect of the Jet Momentum Coefficient}

The injection slot size, suction slot size, and suction surface translation are also fixed to $0.65 \%$ chord, $1.33 \%$ chord, and $1.43 \%$ chord, respectively. The suction location is also fixed to the $80 \%$ chord-wise location from the leading edge. The effects of five jet momentum coefficients, which are $0.02,0.04,0.06,0.08$, and 0.10 respectively, are studied. Two different injection locations, which are $9 \%$ chord-wise location and $1 \%$ chord-wise location from the leading edge, are selected because that the S809 CFJ airfoils with the two injection locations achieve the largest and second largest corrected lift-to-drag ratios when the $C_{\mu}$ is 0.04 .

When the injection location is at $1 \%$ chord-wise location from the leading edge, the aerodynamic coefficients comparisons of S809 CFJ airfoils with various jet momentum coefficients are presented in Figure 13. As shown in Figure 13a,b, the lift and drag characteristics of S809 CFJ airfoil are enhanced with the increased $C_{\mu}$. When the $C_{\mu}$ is 0.02 , the lift coefficient of S809 CFJ airfoil is higher than that of the baseline only at the low AoA, and the S809 CFJ airfoil has the worse lift and drag characteristics at the high AoA. When the $C_{\mu}$ is 0.04 and the AoA is below 8.2 $2^{\circ}$, the lift coefficient of S809 CFJ airfoil is higher than that of the baseline. But when the AoA is higher than $8.2^{\circ}$, the lift coefficient of S809 CFJ airfoil remains almost the same as that of the baseline and the drag coefficient is worse than that of the baseline. While the $C_{\mu}$ is greater than 0.06 , the S809 CFJ airfoil has the higher lift coefficient than the baseline almost at each AoA. The maximum lift coefficients are improved by $9.0 \%, 44.7 \%$, and $67.4 \%$ respectively when the $C_{\mu}$ is $0.06,0.08$, and 0.10 . As shown in Figure 13c, the power consumption coefficient is first decreased and then increased with the increased AoA for each $C_{\mu}$. Moreover, as the $C_{\mu}$ increases, the power consumption coefficient increases at the same small AoA, and the relative increment in the power consumption of S809 CFJ airfoil is larger than the relative increment in the lift coefficient. For example, at $2.05^{\circ} \mathrm{AoA}$, the power consumption coefficient of $\mathrm{S} 809 \mathrm{CFJ}$ airfoil with $0.08 C_{\mu}$ is 2.7 times higher than that with $0.04 C_{\mu}$, while the lift coefficient with $0.08 C_{\mu}$ is only $13.2 \%$ higher than that with $0.04 C_{\mu}$. Thus, as shown in Figure $13 \mathrm{~d}$, the greater the $C_{\mu}$, the lower the corrected lift-to-drag ratio at the low AoA. However, at the high AoA, 
the $\mathrm{S} 809 \mathrm{CFJ}$ airfoil with small $C_{\mu}$ also has a large power consumption coefficient, but most of the energy is converted into heat and is dissipated, while the S809 CFJ airfoil with high $C_{\mu}$ can effectively overcome the flow separation and has the higher lift coefficient. Thus, the greater the $C_{\mu}$, the greater the corrected lift-to-drag ratio at the high AoA. Because of the above reasons, the AoA at the maximum corrected lift-to-drag ratio increases with the increased $C_{\mu}$. The maximum corrected lift-to-drag ratios are improved by $10.4 \%, 34.3 \%$, $33.4 \%, 22.5 \%$, and $8.7 \%$ respectively when the $C_{\mu}$ is $0.02,0.04,0.06,0.08$, and 0.10 . When the $\mathrm{C}_{\mu}$ is 0.04 and 0.06 , the $\mathrm{S} 809 \mathrm{CFJ}$ airfoil has the maximum corrected lift-to-drag ratios.

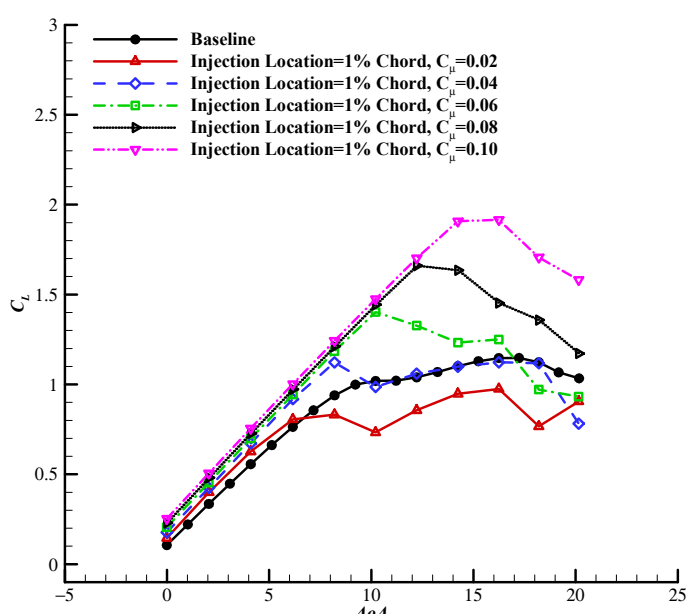

(a)

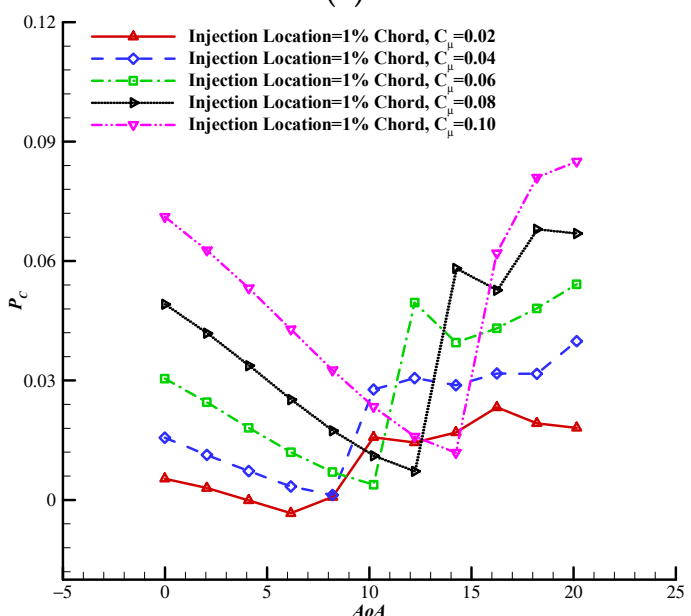

(c)

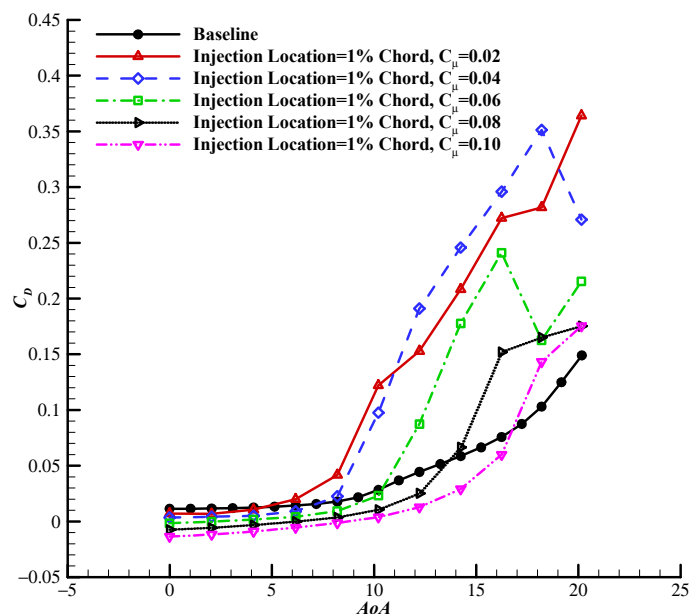

(b)

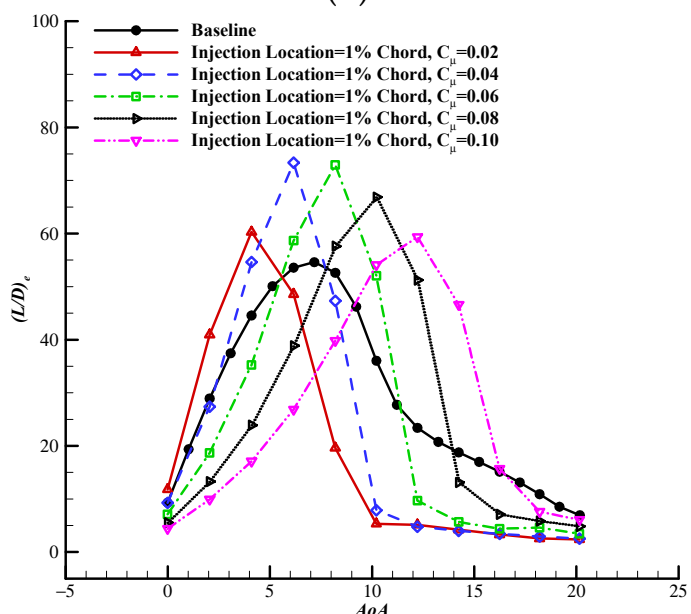

(d)

Figure 13. Aerodynamic coefficients comparisons of the S809 CFJ airfoils with various jet momentum coefficients (injection location $=1 \%$ chord). (a) Lift coefficient; (b) drag coefficient; (c) power coefficient; (d) corrected lift-to-drag ratio.

Although the $\mathrm{S} 809 \mathrm{CFJ}$ airfoil with a given $C_{\mu}$ has the higher maximum corrected lift-to-drag ratio than the baseline, the $S 809 \mathrm{CFJ}$ airfoil with small $C_{\mu}$ has the lower corrected lift-to-drag ratio than the baseline at the high AoA and the $\mathrm{S} 809 \mathrm{CFJ}$ airfoil with high $C_{\mu}$ has the lower corrected lift-to-drag ratio than the baseline at the low AoA. Thus, to ensure that the $\mathrm{S} 809 \mathrm{CFJ}$ airfoil has the higher corrected lift-to-drag ratio and lift coefficient than the baseline at all AoAs, a S809 CFJ strategy with the adaptive $C_{\mu}$ is proposed. The small $C_{\mu}$ is used at the low AoA, and the $C_{\mu}$ gradually increases as the AoA rises. Therefore, based on the performance of S809 CFJ airfoil at different $C_{\mu}$, the $C_{\mu} \mathrm{s}$ of S809 CFJ airfoil with the $1 \%$ chord-wise injection location at various AoAs are tabulated in Table 4. Figure 14 shows the lift coefficients and lift-to-drag ratios of $\mathrm{S} 809 \mathrm{CFJ}$ airfoil with different $C_{\mu}$ at various AoAs. As shown in Figure 14, the lift coefficient and the corrected lift-to-drag ratio of S809 
CFJ airfoil with the adaptive $C_{\mu}$ strategy are comprehensively improved when compared with the baseline, and the maximum lift coefficient and maximum lift-to-drag ratio are improved by $67.4 \%$ and $34.3 \%$, respectively.

Table 4. Jet momentum coefficients of the S809 CFJ airfoil at various AoAs (injection location = $1 \%$ chord).

\begin{tabular}{cc}
\hline AoA & $C_{\mu}$ \\
\hline below $6.16^{\circ}$ & 0.02 \\
\hline $6.16^{\circ}$ & 0.04 \\
\hline $8.20^{\circ}$ & 0.06 \\
\hline $10.21^{\circ}$ & 0.08 \\
\hline above $12.22^{\circ}$ & 0.10 \\
\hline
\end{tabular}

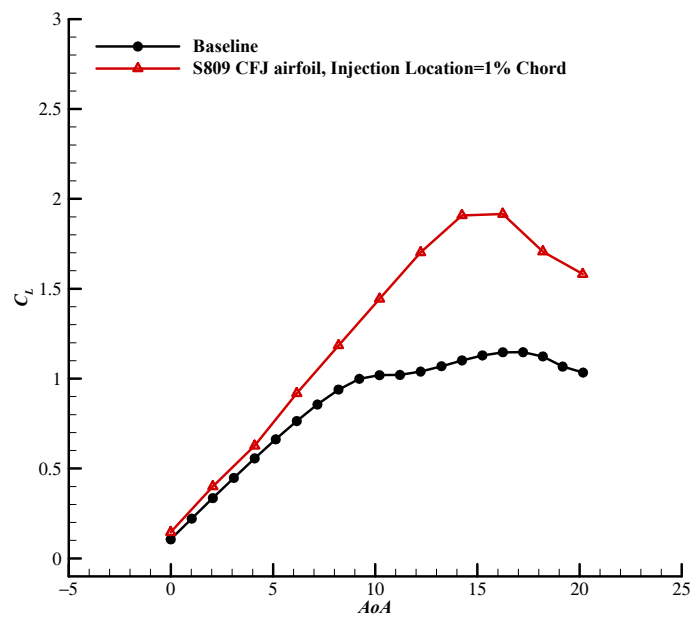

(a)

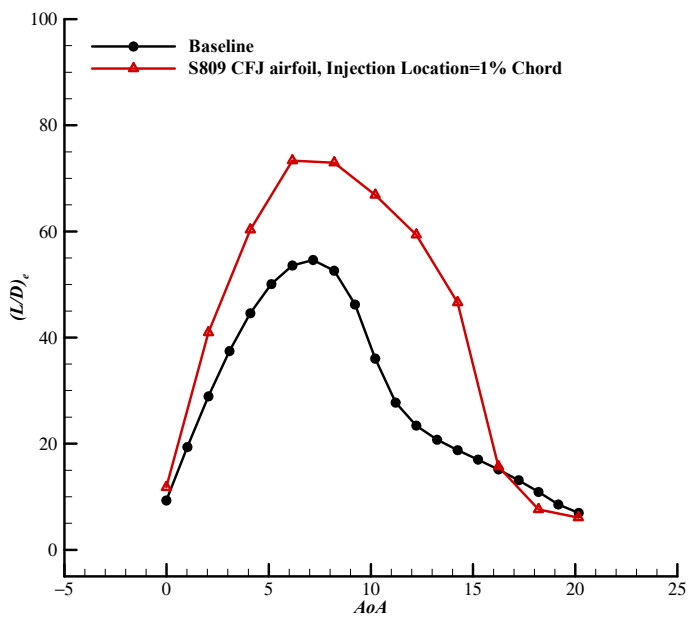

(b)

Figure 14. Aerodynamic coefficients of the S809 CFJ airfoils with different jet momentum coefficients at various AoAs (injection location $=1 \%$ chord). (a) Lift coefficient; (b) corrected lift-to-drag ratio.

In order to further verify the above conclusions and CFJ strategy, the effect of $C_{\mu}$ on the performance of S809 CFJ airfoil is studied when the injection location is at $9 \%$ chord-wise location from the leading edge. Figure 15 shows aerodynamic coefficients comparisons of S809 CFJ airfoils with various jet momentum coefficients. Similarly, the lift and drag characteristics of S809 CFJ airfoil with the 9\% chord-wise injection location are enhanced with the increased $C_{\mu}$. As the $C_{\mu}$ increases, the power consumption coefficient increases at the same small AoA. Moreover, the greater the $C_{\mu}$, the lower the corrected lift-to-drag ratio at the low AoA and the larger the corrected lift-to-drag ratio at the high AoA. At the same time, there are some differences in the aerodynamic characteristics of S809 CFJ airfoil with the $9 \%$ chord-wise injection location and with the $1 \%$ chord-wise injection location. When the $C_{\mu}$ is greater than 0.04 , the S809 CFJ airfoil with the $9 \%$ chordwise injection location has the higher lift coefficient than the S809 CFJ airfoil with the $1 \%$ chord-wise injection location. The maximum lift coefficient increment of S809 CFJ airfoil with the $9 \%$ chord-wise injection location using a given $C_{\mu}$ is greater than that with the $1 \%$ chord-wise injection location. The maximum lift coefficients of S809 CFJ airfoil with the $9 \%$ chord-wise injection location are improved by $84.8 \%, 106.6 \%$, and $119.7 \%$ respectively when compared with that of the baseline when the $C_{\mu}$ is $0.06,0.08$, and 0.10 . As the AoA increases, the power consumption coefficient of S809 CFJ airfoil with the $9 \%$ chord-wise injection location decreases lower than that with the $1 \%$ chord-wise injection location. Therefore, at the high AoA and high $C_{\mu}$, the S809 CFJ airfoil with the 9\% chord-wise injection location 
consumes more energy, which shows that the maximum corrected lift-to-drag ratios of S809 CFJ airfoils with the 0.08 and $0.10 C_{\mu}$ are $0.4 \%$ and $20.6 \%$ lower than that of the baseline. Thus, it is necessary to explore the effect of the injection location.

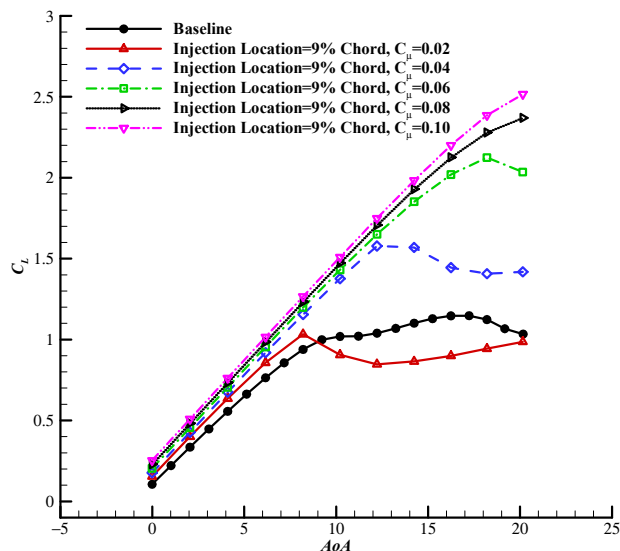

(a)

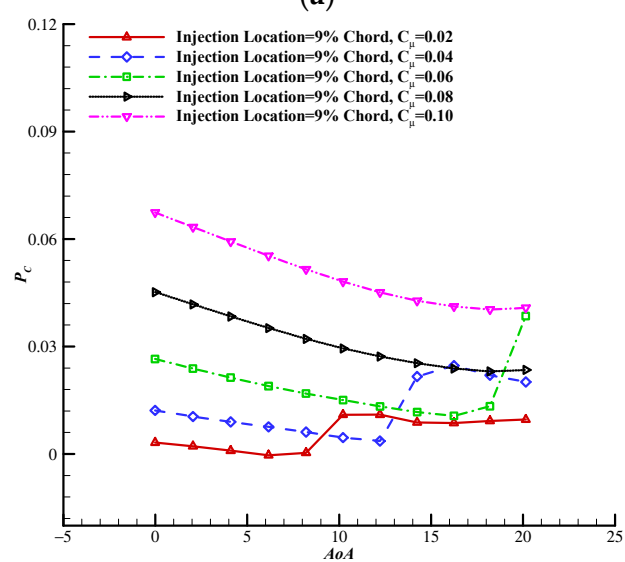

(c)

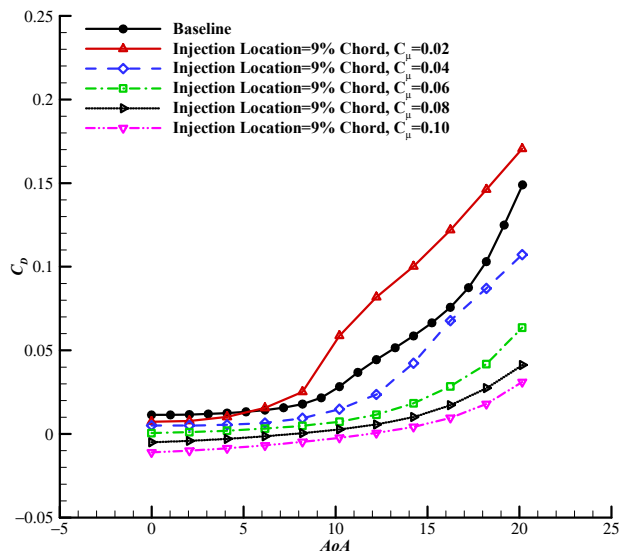

(b)

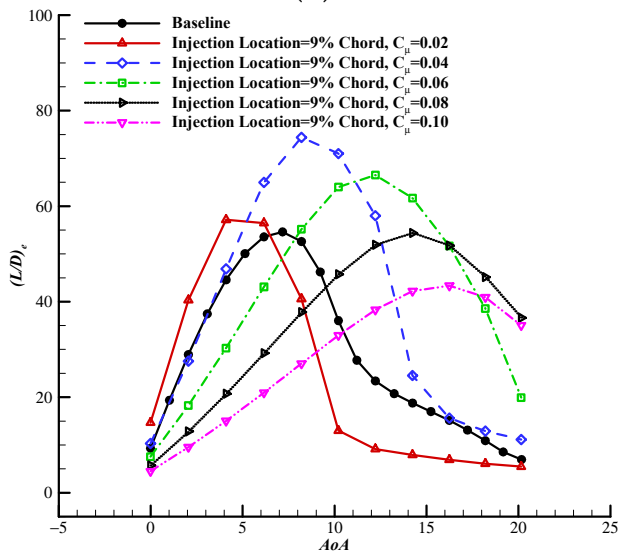

(d)

Figure 15. Aerodynamic coefficients comparisons of the S809 CFJ airfoils with various jet momentum coefficients (injection location $=9 \%$ chord). (a) Lift coefficient; (b) drag coefficient; (c) power coefficient; (d) corrected lift-to-drag ratio.

Figure $15 \mathrm{~d}$ shows that the same CFJ strategy with the adaptive $C_{\mu}$ can be used for the S809 CFJ airfoil with the $9 \%$ chord-wise injection location in order to achieve the higher corrected lift-to-drag ratio and lift coefficient. Based on the performance of S809 CFJ airfoil with the $9 \%$ chord-wise injection location, the $C_{\mu} \mathrm{s}$ at various AoAs are tabulated in Table 5. Figure 16 presents the lift coefficients and lift-to-drag ratios of S809 CFJ airfoils with different $C_{\mu} \mathrm{s}$ at various AoAs. As shown in Figure 16, the lift coefficient and the corrected lift-to-drag ratio of S809 CFJ airfoil with the 9\% chord-wise injection location using the adaptive $C_{\mu}$ strategy are also comprehensively improved when compared with the baseline, and the maximum lift coefficient and maximum lift-to-drag ratio are improved by $119.7 \%$ and $36.2 \%$, respectively.

Table 5. Jet momentum coefficients of the S809 CFJ airfoil at various AoAs (injection location $=9 \%$ chord).

\begin{tabular}{cc}
\hline AoA & $C_{\mu}$ \\
\hline below $6.16^{\circ}$ & 0.02 \\
between $6.16^{\circ}$ and $10.21^{\circ}$ & 0.04 \\
$12.22^{\circ}$ and $14.24^{\circ}$ & 0.06 \\
above $14.24^{\circ}$ & 0.08 \\
\hline
\end{tabular}




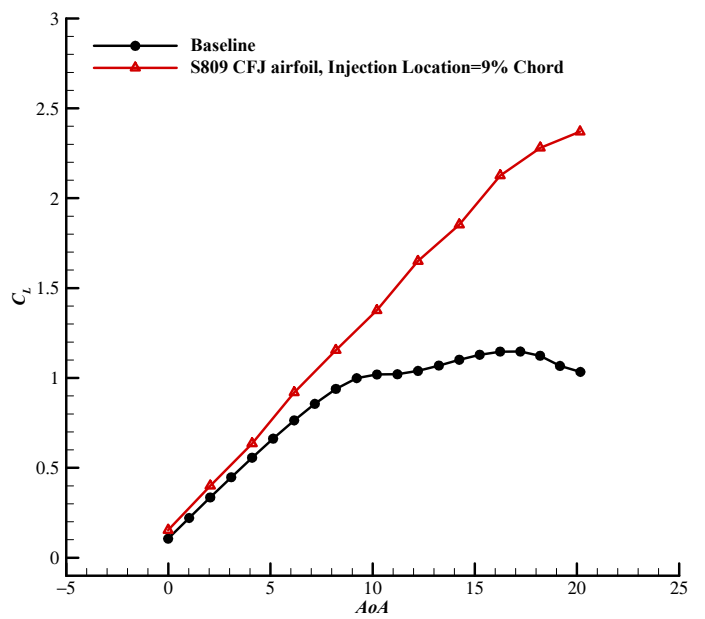

(a)

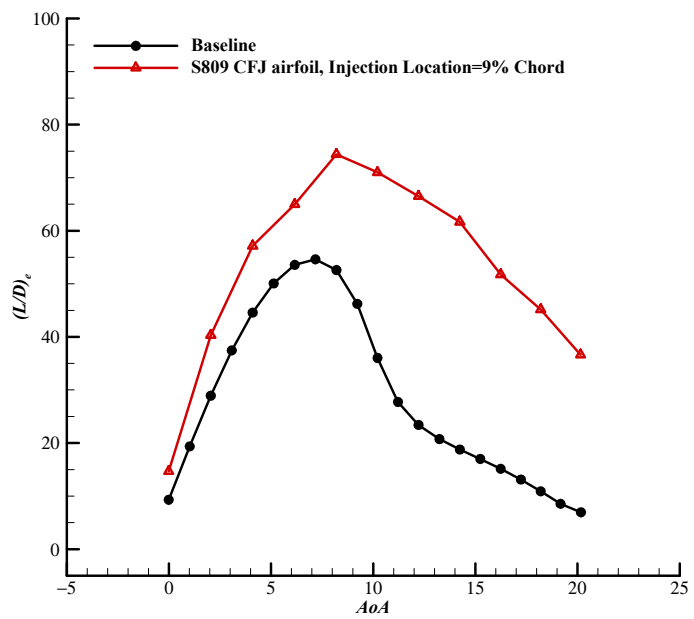

(b)

Figure 16. Aerodynamic coefficients of the S809 CFJ airfoils with different jet momentum coefficients at various AoAs (injection location $=9 \%$ chord). (a) Lift coefficient; (b) corrected lift-to-drag ratio.

\subsection{Performance Enhancement of the Wind Turbine Blade Using the CFJ Technology}

The PROPID code [51], which is a program for the design and analysis of horizontal axis wind turbines based on the blade element momentum theory, is used to evaluate the effect of CFJ technology on the wind turbine blade. The baseline is the PHASE VI wind turbine blade [52] using the $\$ 809$ airfoil as the blade element, and S809 airfoils are replaced by $\mathrm{S} 809 \mathrm{CFJ}$ airfoils with the $9 \%$ chord-wise injection location to construct the CFJ blade. The adaptive $C_{\mu}$ strategy is applied to $S 809 \mathrm{CFJ}$ airfoils and the power consumption of the CFJ technology is included. The radius of the hub and blade are $0.508 \mathrm{~m}$ and $5.029 \mathrm{~m}$, respectively. The chord and twist distributions of the blade are shown in Figure 17.

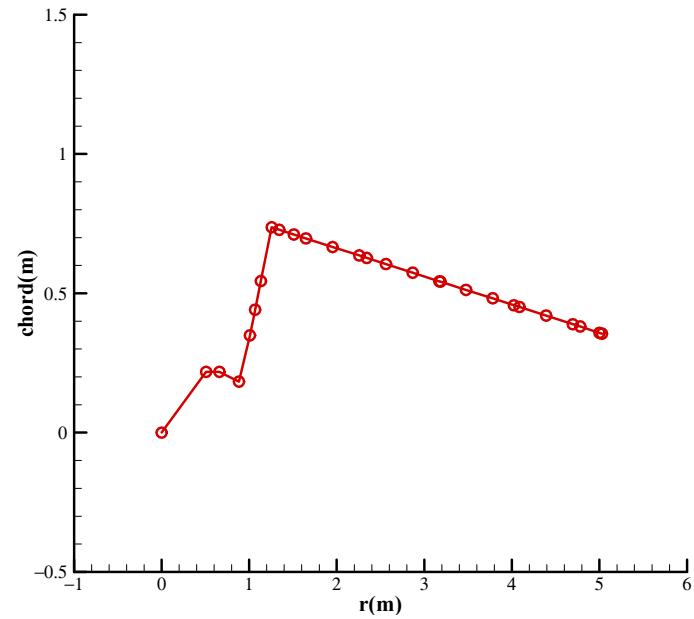

(a)

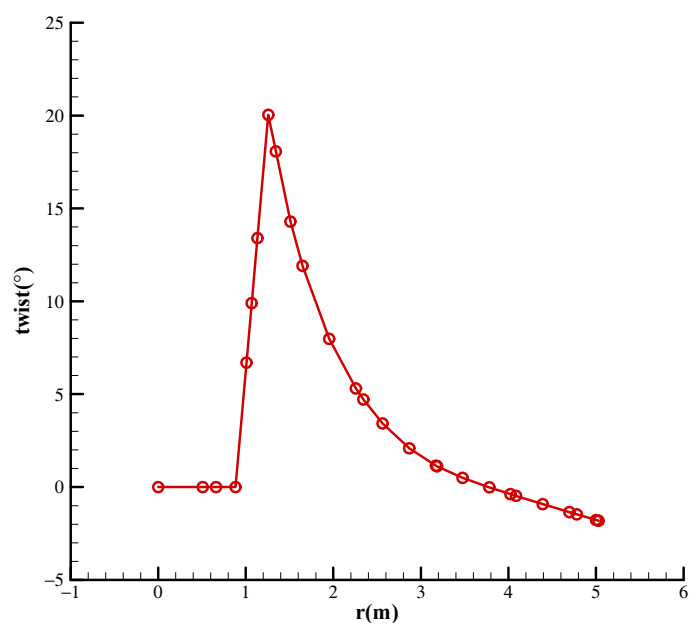

(b)

Figure 17. Chord and twist distributions of the wind turbine blade. (a) Chord distribution; (b) twist distribution.

Figure 18 shows the power coefficients of the CFJ blade and baseline, while the experimental results are from Reference [53]. The power coefficients of the baseline predicted by PROPID code at various tip speed ratios (denoted as $\lambda$ ) are in good agreement with the experimental results, which verifies the reliability of the PROPID code. When $\lambda$ is greater than 7, the power coefficient of the CFJ blade is slightly higher than that of the baseline. However, when $\lambda$ is less than 7 , the power coefficient of the CFJ blade is obviously higher 
than that of the baseline. Table 6 shows the power coefficient comparisons of the CFJ blade and baseline at several tip speed ratios. Although the maximum power coefficient of the CFJ blade only increased by $4.5 \%$ when compared with the baseline, the power coefficients of the CFJ blade are $226.7 \%, 202.9 \%$, and $74.7 \%$ higher than those of the baseline, respectively. Thus, the performance of the Phase VI wind turbine blade is significantly enhanced by using the CFJ technology.

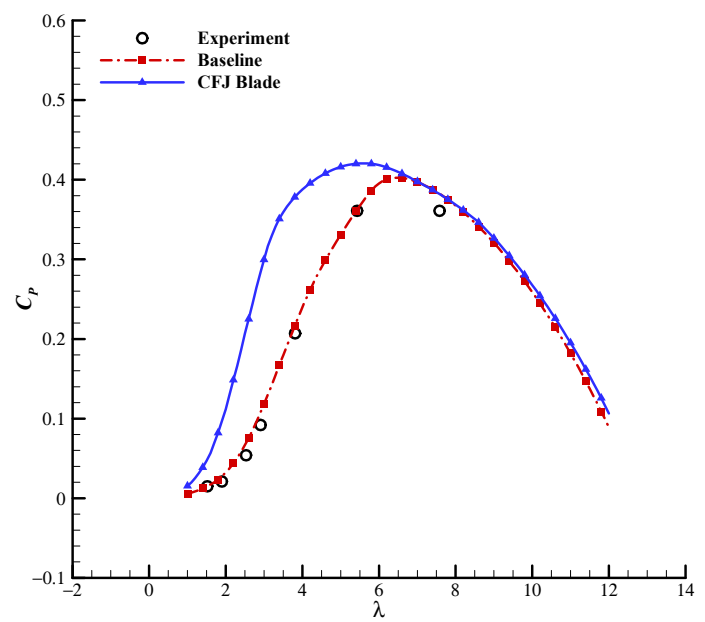

Figure 18. Power coefficient comparisons of the CFJ blade and baseline.

Table 6. Power coefficient comparisons of the CFJ blade and baseline at typical tip speed ratios.

\begin{tabular}{cccc}
\hline $\boldsymbol{\lambda}$ & Baseline & CFJ Blade & Increment \\
\hline 1.52 & 0.015 & 0.049 & $226.7 \%$ \\
2.53 & 0.070 & 0.212 & $202.9 \%$ \\
3.81 & 0.217 & 0.379 & $74.7 \%$ \\
7.58 & 0.382 & 0.382 & $0.0 \%$ \\
\hline
\end{tabular}

\section{Conclusions}

In this study, the effects of the injection location and jet momentum coefficient are studied, and an adaptive jet momentum coefficient strategy of the CFJ technology is proposed. Meanwhile, the effects of the CFJ technology on the performance enhancement of the Phase VI wind turbine blade are explored. Some major conclusions can be summarized as follows:

(1) The CFJ technology can significantly improve the maximum lift coefficient and maximum corrected lift-to-drag ratio of the S809 airfoil;

(2) At the low AoA, the closer the injection location is to the leading edge, the better the lift and drag characteristics of S809 CFJ airfoil. At the high AoA, the more downstream the injection location, the stronger the flow separation suppression capability of S809 CFJ airfoil;

(3) A S809 CFJ strategy with the adaptive $C_{\mu}$ is proposed. The small $C_{\mu}$ is used at the low AoA, and the $C_{\mu}$ gradually increases as the AoA rises. The performance of S809 CFJ airfoil with adaptive $C_{\mu}$ is comprehensively enhanced, and the maximum lift coefficient and maximum corrected lift-to-drag ratio of the typical S809 CFJ airfoil are improved by $119.7 \%$ and $36.2 \%$, respectively;

(4) The application of the CFJ technology to the Phase VI wind turbine blade can greatly improve the power coefficient at the low tip speed ratio. The maximum power coefficient of CFJ blade can be increased by $4.5 \%$, and the power coefficient of CFJ blade can be boosted by $226.7 \%$ when the tip speed ratio is 1.52 . 


\section{Future Work}

The RANS or LES methods should be used to further investigate the effects of the CFJ technology on the performance of the wind turbine blade.

Author Contributions: Conceptualization, X.Y. and B.S.; methodology, S.Z. and X.Y.; validation, S.Z.; formal analysis, X.Y.; investigation, S.Z.; resources, S.Z. and B.S.; data curation, S.Z. and X.Y.; writing-original draft preparation, S.Z.; writing—review and editing, S.Z. and B.S.; visualization, S.Z.; supervision, X.Y. and B.S.; project administration, X.Y. and B.S.; funding acquisition, X.Y. All authors have read and agreed to the published version of the manuscript.

Funding: This research was funded by NATIONAL NATURAL SCIENCE FOUNDATION OF CHINA, grant number 11672243.

Institutional Review Board Statement: Not applicable.

Informed Consent Statement: Not applicable.

Data Availability Statement: Not applicable.

Conflicts of Interest: The authors declare no conflict of interest.

\section{References}

1. World Wind Energy Association. Available online: https:/ /wwindea.org/worldwide-wind-capacity-reaches-744-gigawatts / (accessed on 24 March 2021).

2. Ahmed, M.R.; Nabolaniwaqa, E. Performance Improvement of a Wind Turbine Blade Designed for Low Wind Speeds With a Passive Trailing Edge Flap. In Proceedings of the ASME 2012 International Mechanical Engineering Congress \& Exposition, Houston, TX, USA, 9-15 November 2012.

3. Fernandez-Gamiz, U.; Zulueta, E.; Boyano, A.; Ansoategui, I.; Uriarte, I. Five Megawatt Wind Turbine Power Output Improvements by Passive Flow Control Devices. Energies 2017, 10, 742. [CrossRef]

4. Zhang, H.; Zhao, Z.D.; Zhou, G.X.; Kang, S. Experimental Investigation of the Effect of Gurney Flap on Du93-W-210 Airfoil Aerodynamics Performance. Acta Energy Sol. Sin. 2017, 38, 601-606.

5. Ahmed, M.R.; Nabolaniwaqa, E. Performance Studies on a Wind Turbine Blade Section for Low Wind Speeds With a Gurney Flap. J. Energy Resour. Technol. 2019, 141, 111202. [CrossRef]

6. Inigo, A.; Unai, F.G.; Ekaitz, Z.; Aitor, S.A.; Daniel, S.A. Parametric Study of a Gurney Flap Implementation in a Du91w(2)250 Airfoil. Energies 2019, 12, 294.

7. Afjeh, A.A.; Keith, T.G.; Fateh, A. Predicted aerodynamic performance of a horizontal-axis wind turbine equipped with vortex generators. J. Wind. Eng. Ind. Aerodyn. 1990, 33, 515-529. [CrossRef]

8. Dai, L.P.; Jiao, J.D.; Li, X.K.; Kang, S.; Zhao, P. Numerical Investigation on Effect of Installation Position of Vortex Generator on Aerodynamic Characteristics of Wind Turbine Airfoil. Acta Energy Sol. Sin. 2016, 37, 276-281.

9. Wang, H.; Zhang, B.; Qiu, Q.; Xu, X. Flow control on the NREL S809 wind turbine airfoil using vortex generators. Energy 2017, 118, 1210-1221. [CrossRef]

10. Li, X.; Yang, K.; Wang, X. Experimental and Numerical Analysis of the Effect of Vortex Generator Height on Vortex Characteristics and Airfoil Aerodynamic Performance. Energies 2019, 12, 959. [CrossRef]

11. Ye, Q.; Avallone, F.; Van Der Velden, W.; Casalino, D. Effect of Vortex Generators on NREL Wind Turbine: Aerodynamic Performance and Far-Field Noise. J. Phys. Conf. Ser. 2020, 1618, 52077. [CrossRef]

12. Khameneh, N.; Tadjfar, M. Improment of Wind Turbine Efficiency by Using Synthetic Jets. In Proceedings of the ASME 2016 Fluids Engineering Division Summer Meeting, Washington, DC, USA, 10-14 July 2016.

13. Moshfeghi, M.; Hur, N. Numerical study on the effects of a synthetic jet actuator on S809 airfoil aerodynamics at different flow regimes and jet flow angles. J. Mech. Sci. Technol. 2017, 31, 1233-1240. [CrossRef]

14. Ali, B.; Ouahiba, G.; Hamid, O.; Ahmed, B. Aerodynamic Optimization of Active Flow Control over S809 Airfoil Using Synthetic Jet. In Proceedings of the 2018 International Conference on Wind Energy and Applications in Algeria, Algiers, Algeria, 6-7 November 2018.

15. Zhu, H.; Hao, W.; Li, C.; Ding, Q. Simulation on flow control strategy of synthetic jet in an vertical axis wind turbine. Aerosp. Sci. Technol. 2018, 77, 439-448. [CrossRef]

16. Maldonado, V.; Gupta, S. Increasing the power efficiency of rotors at transitional Reynolds numbers with synthetic jet actuators. Exp. Therm. Fluid Sci. 2019, 105, 356-366. [CrossRef]

17. Xu, H.-Y.; Qiao, C.-L.; Yang, H.-Q.; Ye, Z.-Y. Active Circulation Control on the Blunt Trailing Edge Wind Turbine Airfoil. AIAA J. 2018, 56, 554-570. [CrossRef]

18. Xu, H.Y.; Dong, Q.L.; Qiao, C.L.; Ye, Z.Y. Flow Control over the Blunt Trailing Edge of Wind Turbine Airfoils Using Circu-Lation Control. Energies 2018, 11, 619. [CrossRef] 
19. Aono, H.; Nonomura, T.; Yakeno, A.; Fujii, K. Plasma Flow Control Simulation of an Airfoil of Wind Turbine at an Inter-Mediate Reynolds Number. In Proceedings of the ASME 2013 Fluids Engineering Division Summer Meeting, Incline Village, NV, USA, 7-11 July 2013.

20. Baleriola, S.; Leroy, A.; Loyer, S.; Devinant, P.; Aubrun, S. Circulation control on a rounded trailing-edge wind turbine airfoil using plasma actuators. J. Phys. Conf. Ser. 2016, 753, 52001. [CrossRef]

21. Li, G.Q.; Zhang, W.G.; Jiang, Y.B.; Yang, P.Y. Experimental Investigation of Dynamic Stall Flow Control for Wind Turbine Airfoils Using a Plasma Actuator. Energy 2019, 185, 90-101.

22. Li, G.Q.; Yi, S.H. Large Eddy Simulation of Dynamic Stall Flow Control for Wind Turbine Airfoil using Plasma Actuator. Energy 2020, 212, 118753.

23. Aono, H.; Fukumoto, H.; Abe, Y.; Sato, M.; Nonomura, T.; Fujii, K. Separated Flow Control of Small Horizontal-Axis Wind Turbine Blades Using Dielectric Barrier Discharge Plasma Actuators. Energies 2020, 13, 1218. [CrossRef]

24. Fadaei, M.; Davari, A.; Sabetghadam, F.; Soltani, M. Enhancement of a Horizontal Axis Wing Turbine Airfoil Performance Using Single Dielectric Barrier Discharge Plasma Actuator. Proc. IMechE Part A J. Power Energy 2021, 235, 476-493. [CrossRef]

25. Zhu, H.; Hao, W.; Li, C.; Ding, Q.; Wu, B. Application of flow control strategy of blowing, synthetic and plasma jet actuators in vertical axis wind turbines. Aerosp. Sci. Technol. 2019, 88, 468-480. [CrossRef]

26. Zha, G.-C.; Paxton, C. A Novel Airfoil Circulation Augment Flow Control Method Using Co-Flow Jet. In Proceedings of the 2rd AIAA Flow Control Conference, Portland, OR, USA, 28 June-1 July 2004.

27. Zha, G.C.; Gao, W.; Paxton, C. Numerical Simulation of Co-Flow Jet Airfoil Flows. In Proceedings of the 44th AIAA Aerospace Sciences Meeting and Exhibit, Reno, NV, USA, 9-12 January 2006.

28. Zha, G.-C.; Carroll, B.F.; Paxton, C.D.; Conley, C.A.; Wells, A. High-Performance Airfoil Using Coflow Jet Flow Control. AIAA J. 2007, 45, 2087-2090. [CrossRef]

29. Yang, Y.C.; Zha, G.C. Super-Lift Coefficient of Active Flow Control Airfoil: What is the Limit? In Proceedings of the 55th AIAA Aerospace Sciences Meeting, Grapevine, TX, USA, 9-13 January 2017.

30. Yang, Y.C.; Fernandez, M.; Zha, G.C. Improved Delayed Detached Eddy Simulation of Super-Lift Flow of Co-Flow Jet Airfoil. In Proceedings of the 2018 AIAA Aerospace Sciences Meeting, Kissimmee, FL, USA, 8-12 January 2018.

31. Yang, Y.C.; Zha, G.C. Super Lift Coefficient of Cylinder Using Co-Flow Jet Active Flow Control. In Proceedings of the 2018 AIAA Aerospace Sciences Meeting, Kissimmee, FL, USA, 8-12 January 2018.

32. Yang, Y.C.; Zha, G.C. Numerical Investigation of Ultra-High Lift Coefficient Co-Flow Jet Wing without Flaps. In Proceedings of the 2018 AIAA Aerospace Sciences Meeting, Kissimmee, FL, USA, 8-12 January 2018.

33. Zha, G.; Yang, Y.; Ren, Y.; McBreen, B. Super-Lift and Thrusting Airfoil of Coflow Jet Actuated by Micro-Compressors. In Proceedings of the 2018 Flow Control Conference, Atlanta, GA, USA, 25-29 June 2018.

34. Ren, Y.; Zha, G. Simulation of 3D Co-Flow Jet Airfoil with Embedded Micro-Compressor Actuator. In Proceedings of the 2018 AIAA Aerospace Sciences Meeting, Kissimmee, FL, USA, 8-12 January 2018.

35. Ren, Y.; Zha, G. Design of Injection and Suction Ducts for Co-Flow Jet Airfoils with Embedded Micro-Compressor Actuator. In Proceedings of the 2018 Flow Control Conference, Atlanta, GA, USA, 25-29 June 2018.

36. Ren, Y.; Zha, G. Design of Injection Jet Span Profile for Co-Flow Jet Airfoil. In Proceedings of the AIAA Scitech 2019 Forum, San Diego, CA, USA, 7-11 January 2019.

37. Zhang, S.L.; Yang, X.D.; Song, B.F.; Xu, J.H. Numerical and Experimental Study of the Co-Flow Jet Airfoil Performance Enhancement. In Proceedings of the 55th AIAA Aerospace Sciences Meeting, Grapevine, TX, USA, 9-13 January 2017.

38. Zhu, M.; Yang, X.D.; Song, C.; Song, W.P. High Synergy Method for Near Space Propeller Using Co-Flow Jet Control. Acta Aeronaut. Astronaut. Sin. 2014, 35, 1549-1559.

39. Yang, X.-D.; Jiang, W.-R.; Zhang, S.-L. Analysis of Co-Flow Jet Effect on Dynamic Stall Characteristics Applying to Rotor Airfoils. IOP Conf. Ser. Mate. Sci. Eng. 2019, 491, 12010. [CrossRef]

40. Zha, G.-C.; Paxton, C.D.; Conley, C.A.; Wells, A.; Carroll, B.F.; Wells, A. Effect of Injection Slot Size on the Performance of Coflow Jet Airfoil. J. Aircr. 2006, 43, 987-995. [CrossRef]

41. Wang, B.; Haddoukessouni, B.; Lévy, J.; Zha, G.-C. Numerical Investigations of Injection-Slot-Size Effect on the Performance of Coflow Jet Airfoils. J. Aircr. 2008, 45, 2084-2091. [CrossRef]

42. Lefebvre, A.M.; Zha, G. Co-Flow Jet Airfoil Trade Study Part I: Energy Consumption and Aerodynamic Efficiency. In Proceedings of the 32nd AIAA Applied Aerodynamics Conference, Atlanta, GA, USA, 16-20 June 2014.

43. Lefebvre, A.; Zha, G.C. Co-Flow Jet Airfoil Trade Study Part II: Moment and Drag. In Proceedings of the 32nd AIAA Applied Aerodynamics Conference, Atlanta, GA, USA, 16-20 June 2014.

44. Xu, J.H.; Li, K.; Song, W.P.; Yang, X.D. Influence of Co-Flow Jet Key Parameters on Airfoil Aerodynamic Performance at Low Reynolds Number. Acta Aeronaut. Astronaut. Sin. 2018, 39, 122018.

45. Xu, H.-Y.; Xing, S.-L.; Ye, Z.-Y. Numerical study of the $\mathrm{S} 809$ airfoil aerodynamic performance using a co-flow jet active control concept. J. Renew. Sustain. Energy 2015, 7, 23131. [CrossRef]

46. Xu, H.-Y.; Qiao, C.-L.; Ye, Z.-Y. Dynamic Stall Control on the Wind Turbine Airfoil via a Co-Flow Jet. Energies 2016, 9 , 429. [CrossRef]

47. Sun, X.; Xu, Y.; Huang, D. Numerical simulation and research on improving aerodynamic performance of vertical axis wind turbine by co-flow jet. J. Renew. Sustain. Energy 2019, 11, 13303. [CrossRef] 
48. Xu, K.W.; Zha, G.C. Investigation of Coflow Jet Active Flow Control for Wind Turbine Airfoil. In Proceedings of the AIAA AVIATION 2020 FORUM, Virtual Online, 15-19 June 2020.

49. Xu, K.; Zha, G. High Efficiency Wind Turbine Using Co-Flow Jet Active Flow Control. In Proceedings of the ASME Turbo Expo 2021, Virtual Online, 7-11 June 2021.

50. Somers, D.M. Design and Experimental Results for the S809 Airfoil; Technical Report NREL/SR-440-6918; National Renewable Energy Laboratory (NREL): Golden, CO, USA, 1997.

51. PROPID for Horizontal Axis Wind Turbine Design. Available online: https://m-selig.ae.illinois.edu/propid.html (accessed on 9 May 2021).

52. Hand, M.M.; Simms, D.A.; Fingersh, L.J.; Jager, D.W.; Cotrell, J.R.; Schreck, S.; Larwood, S.M. Unsteady Aerodynamics Experiment Phase VI: Wind Tunnel Test Configurations and Available Data Campaigns; Technical Report NREL/TP-500-29955; National Renewable Energy Laboratory (NREL): Golden, CO, USA, 2001.

53. Sørensen, N.N.; Michelsen, J.A.; Schreck, S. Navier-Stokes predictions of the NREL phase VI rotor in the NASA Ames $80 \mathrm{ft} \times 120 \mathrm{ft}$ wind tunnel. Wind. Energy 2002, 5, 151-169. [CrossRef] 